\title{
Plasticity in AAA+ proteases reveals ATP-dependent substrate specificity principles
}

\author{
Samar A. Mahmoud ${ }^{1,2}$, Berent Aldikacti ${ }^{1,2}$, Peter Chien ${ }^{1,2^{*}}$
}

$21 \quad{ }^{1}$ Department of Biochemistry and Molecular Biology, ${ }^{2}$ Molecular and Cellular Biology 22 Program, University of Massachusetts Amherst, Amherst, MA 01003, USA.

23
Corresponding Author:

Peter Chien

240 Thatcher Rd, Amherst, MA 01003

pchien@umass.edu

(413) 545-2310 

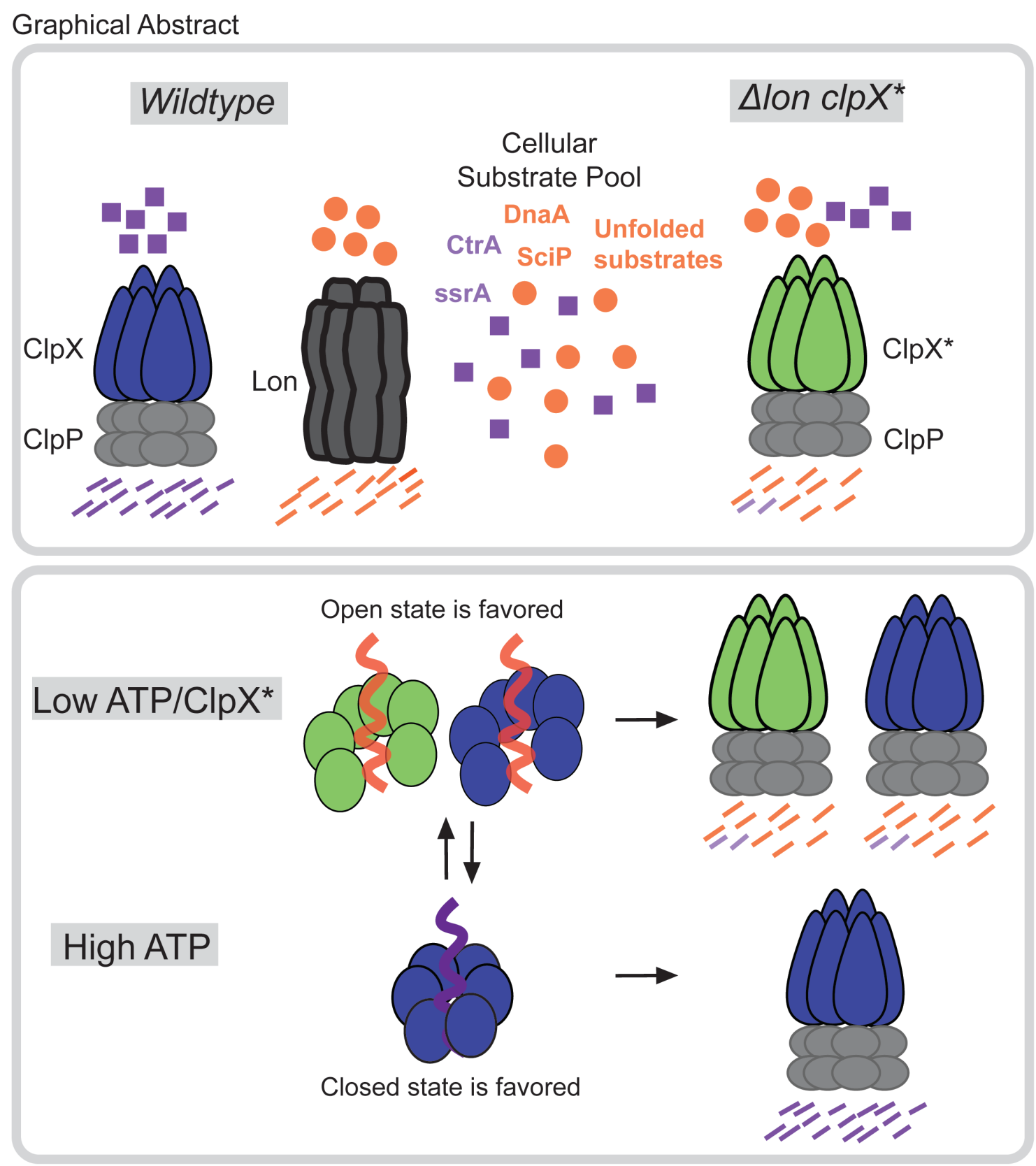

In a wildtype cell, AAA+ proteases Lon and CIpXP promote normal growth by degrading distinct substrates. ClpX*P can compensate for the absence of the Lon protease by tuning CIpXP substrate specificity to better degrade Lon-privileged substrates (such as DnaA, SciP, and misfolded proteins) but this comes at the cost of native CIpXP substrates (such as ssrA-tagged proteins and CtrA). We propose that ClpX alternates between a closed and open conformation and promoting one state over the other leads to alterations in substrate specificity. In the presence of ClpX* or in ATP-limited conditions, the open state is favored, allowing capture and recognition of substrates such as casein. The balance shifts to the closed state under high ATP conditions, allowing degradation of substrates such as GFP-ssrA, which preferentially bind the closed state. 


\section{Summary}

In bacteria, AAA+ proteases such as Lon and CIpXP specifically degrade substrates to promote growth and stress responses. Here, we find that an ATP-binding mutant of ClpX suppresses physiological defects of a Lon-deficient strain by shifting CIpXP protease specificity toward normally Lon-restricted substrates and away from normal ClpXP targets. Reconstitution with purified proteins assigns these effects to changes in direct recognition and processing of substrates. We show that wildtype CIpXP specificity can be similarly altered when ATP hydrolysis is reduced, which unexpectedly accelerates degradation of some substrates. This activation corresponds with changes in ClpX conformation, leading to a model where ClpX cycles between 'capture' and 'processive' states depending on ATP loading. Limiting ATP binding alters dynamics between states affording better recognition of unorthodox substrates, but worse degradation of proteins specifically bound by the processive state. Thus, AAA+ protease specificities can be directly tuned by differences in ATP hydrolysis rates.

\section{Highlights}

- A mutation in the Walker B region of CIpX induces recognition of new substrates.

- Proteases are optimized for specific functions but barrier to recognize new substrates is easily overcome.

- Expanding substrate recognition by a protease comes at the cost of reducing native substrate degradation.

- Decreasing ATP enhances CIpXP mediated degradation of certain classes of substrates.

- ClpX adopts distinct conformational states to favor better recognition of some substrates over others.

\section{Introduction}

Energy-dependent protein degradation regulates normal growth and stress responses in all cells. In bacteria, regulated proteolysis is carried out by several energy-dependent $\mathrm{AAA}+($ ATPases associated with cellular activities) proteases which include Lon, ClpXP, 
84 ClpAP, HsIUV, FtsH (Gur et al., 2011; Mahmoud and Chien, 2018). These proteases share a similar general architecture containing an unfoldase domain and a non-specific peptidase (Sauer and Baker, 2011). The two domains can be encoded on a single polypeptide, such as in the case of Lon and $\mathrm{FtsH}$, or they can be encoded by separate proteins, an ATPase, such as CIpX, and a peptidase, such as ClpP (Baker and Sauer, 2012; Langklotz et al., 2012; Lee and Suzuki, 2008). Using the power of ATP hydrolysis, the unfoldase recognizes, unfolds, and translocates substrates into the sequestered peptidase chamber to be degraded. Because they are critical for maintaining proteostasis, loss of these proteases results in defects in growth, cell-cycle progression, stress responses, and pathogenesis (Breidenstein et al., 2012; Howard-Flanders et al., 1964; Jenal and Fuchs, 1998; Rogers et al., 2016).

Despite the similarities in both architecture and mechanism, these proteases generally have distinct niches of substrate preference. For example, in Caulobacter crescentus, Lon is the principal protease for degradation of the replication initiator DnaA (Jonas et al., 2013), the methyltransferase CcrM (Wright et al., 1996), and the transcriptional regulator SciP (Gora et al., 2013). By contrast, the ClpXP protease degrades cell-cycle factors like CtrA and TacA during cell-cycle progression through an adaptor hierarchy

102 (Joshi et al., 2015). Lon is also responsible for misfolded protein degradation

103 (Goldberg, 1972; Gur and Sauer, 2008; Jonas et al., 2013). However, while a handful of

104 target substrates for these AAA+ have been identified, it remains unclear how proteases

105 are able to discriminate among protein targets and recognize distinct substrates for

106 irreversible degradation.

108 In this work, we find that a variant of $\mathrm{ClpX}\left(c / p X^{*}\right)$ can compensate for the absence of 109 the Lon protease. Expression of $c / p X^{\star}$ suppresses defects in motility, growth,

110 filamentation, and sensitivity to stress normally seen in a $\Delta / o n$ strain. In addition to this 111 phenotypic rescue, degradation of normal Lon substrates is restored in vivo and in vitro 112 by $\mathrm{ClpX}^{*} \mathrm{P}$. This increased ability to degrade noncanonical substrates comes at the cost 113 of reduced degradation of ClpXP-specific substrates, resulting in fitness defects in 114 otherwise wildtype strains expressing the $c / p X^{*}$ allele. Further mechanistic 
115 characterization shows that $\mathrm{ClpX}$ * has reduced catalytic efficiency for ATP hydrolysis, 116 suggesting a connection between ATP utilization and substrate specificity. Consistent 117 with this, limiting ATP for wildtype ClpXP shifts substrate preferences to that of $C l p X^{*} P$.

118 Limited proteolysis shows that the ClpX unfoldase adopts distinct conformations under

119 these ATP limiting or saturating conditions. Taken together, our work demonstrates that 120 ATP-dependent dynamics between conformational states are important for substrate 121 recognition by AAA+ unfoldases.

\section{Results}

124 A suppressor screen identifies a clpX mutant which rescues $\Delta$ lon

125 We reasoned that a suppressor screen would allow us to identify novel Lon-related

126 interactions and used a transposon library to identify mutations that restore motility to a

$127 \Delta$ lon strain. We isolated a high motility mutant with a transposon insertion in

128 CCNA_00264; however transduction experiments revealed that this transposon alone 129 was not able to rescue motility (Figure S1A). Sequencing the genome of the suppressor

130 strain showed a point mutation in $c / p X$, which resulted in a single amino acid change

131 from glycine 178 to alanine $\left(c / p X^{*}\right)$. This glycine is highly conserved among ClpX

132 homologs and immediately adjacent to the Walker B motif (Figure S1C). By generating

133 the $c / p X^{*}$ allele in a $\Delta /$ on background, we found that $c / p X^{*}$ on its own partially restores

134 motility (Figure 1A).

136 We were intrigued that a single mutation in clpX could restore motility to cells lacking 137 Lon and chose to further characterize this strain. Cells lacking Lon show growth defects 138 that exhibit as an extended lag phase and reduced cell mass accumulation in stationary 139 phase (Figure 1A). The $c / p X^{*}$ allele rescues the mass accumulation defect, but not the 140 extended lag (Figure 1A). $\Delta$ lon strains also have significant morphological deficiencies

141 including elongated cell length and longer stalks, external polar organelles characteristic 142 of Caulobacter. We found that the $c / p X^{*}$ mutant restored the cell length of $\Delta$ lon strains to 143 near wildtype levels (Figure 1B, 1C). However, stalks remained elongated in the $\Delta / o n$ $144 c / p X^{*}$ strain (Figure 1B, 1C). 
146 We next wondered if, in addition to suppression of $\Delta$ lon morphological and growth

147 defects, sensitivity to certain stressors would be suppressed by this clpX*allele. One of

148 the originally described phenotypes for E. coli lon mutants is DNA damage sensitivity

149 (Mizusawa and Gottesman, 1983; Witkin, 1946) and Caulobacter Alon strains are also

150 highly sensitive to various DNA damaging agents (Zeinert et al., 2018), including

151 mitomycin $\mathrm{C}$ (Figure $1 \mathrm{C}$ ). We found that $\Delta$ lon $c / p X^{*}$ was 100 -fold more resistant to MMC

152 than $\Delta$ lon alone (Figure $1 \mathrm{C}$ ).

154 As mentioned previously, Lon is the major protease responsible for the degradation of

155 the replication initiator DnaA and $\Delta$ lon strains accumulate excess chromosome content

156 (Jonas et al., 2013; Wright et al., 1996). Like the morphological abnormalities,

157 overreplication is suppressed in the $\Delta /$ lon $c / p X^{*}$ strain which shows similar chromosome

158 content as wildtype cells (Figure 1D, S1D). To determine if this rescue extended to a

159 systems-level correction, we compared RNA-seq profiles of wildtype, $\Delta$ lon and $\Delta$ lon

$160 \quad c / p X^{*}$ strains. As expected, many genes (435) are differentially expressed upon loss of

161 Lon (Figure 1E), while the $\Delta$ lon clpX* strain shows fewer differences (119) with only 85

162 genes overlapping between these sets. Our interpretation is that expression of $c / p X^{*}$ in

163 a $\Delta$ lon background largely shifts the phenotypes and transcriptional landscape to more 164 wildtype profiles. 
Figure 1

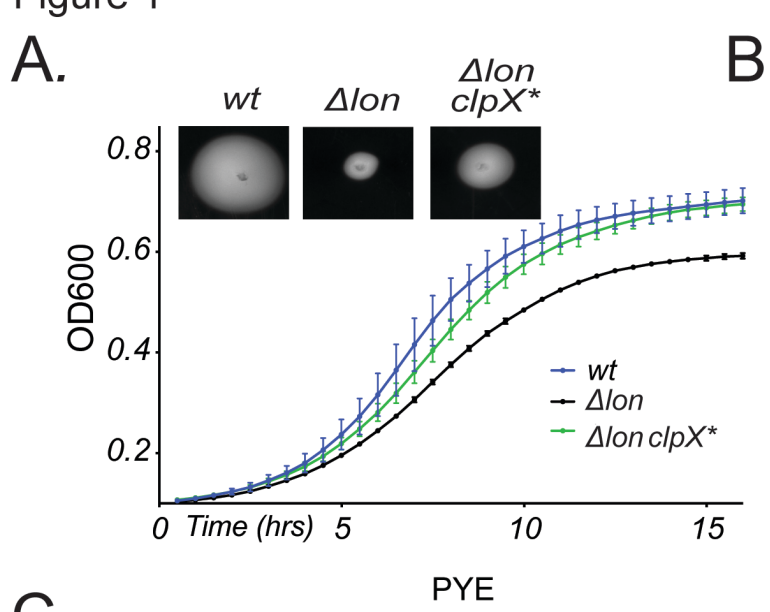

C.

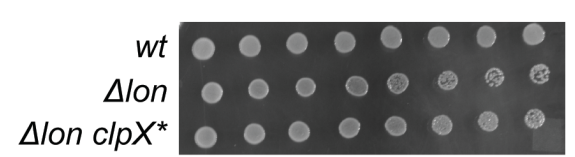

$0.25 \mu \mathrm{g} / \mathrm{mL}$ MMC
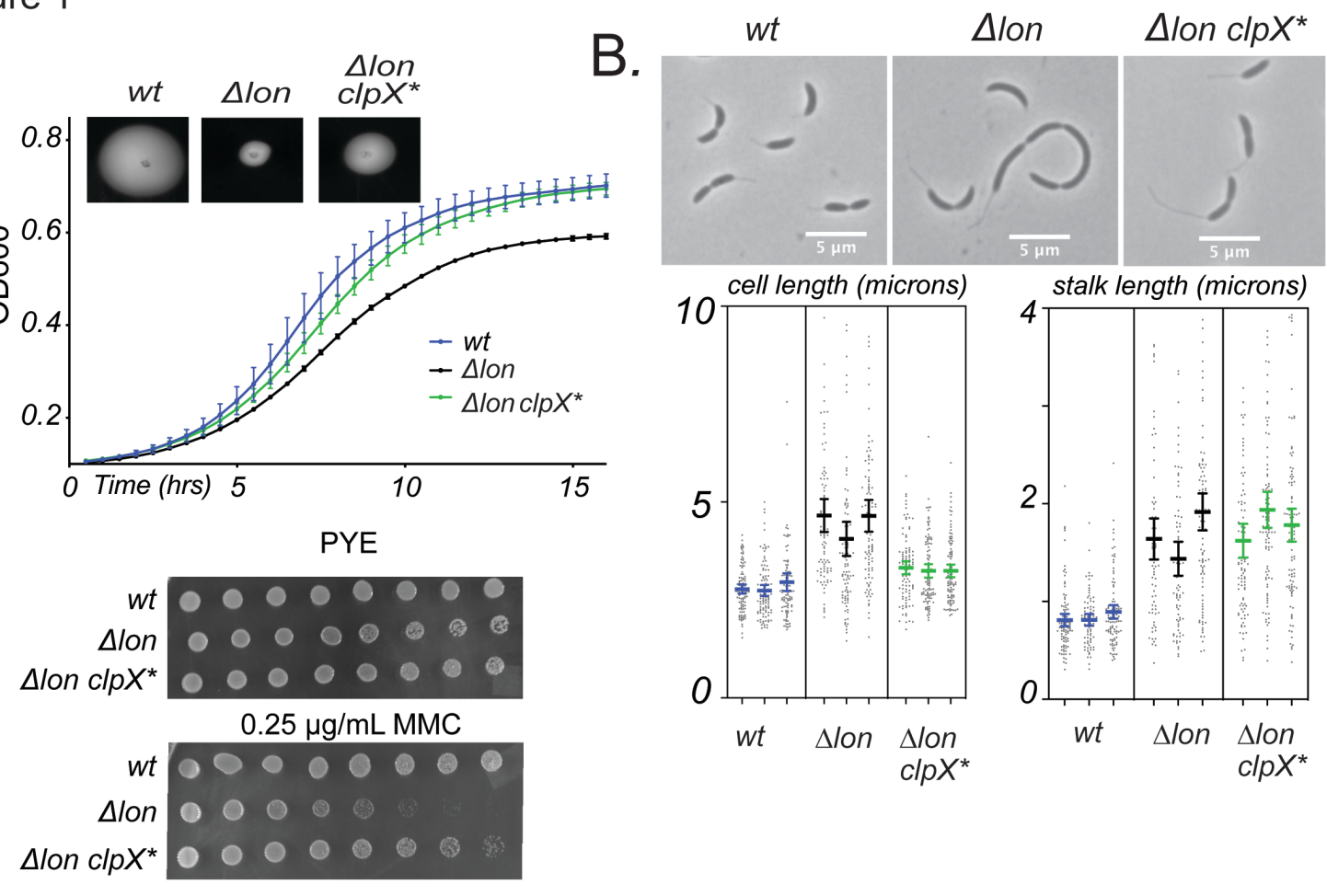

D.

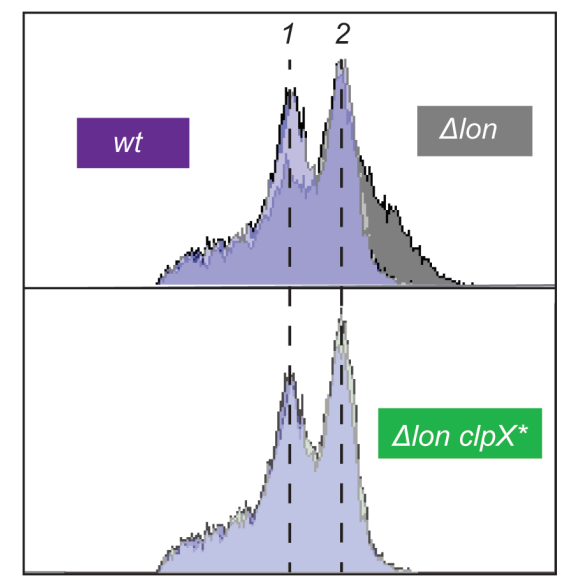

E.

Differentially

Expressed Genes

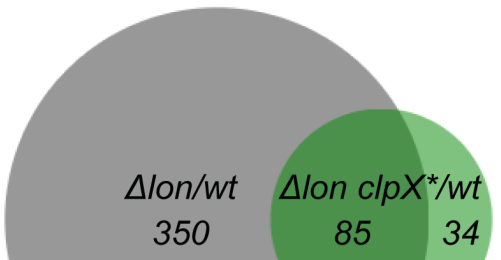

$F D R<0.01$

DNA content

Figure 1: clp $X^{*}$ mutant suppresses $\Delta /$ on phenotypes

167 A. Growth curves of wild type (wt), $\Delta / o n$, and $\Delta / o n c / p X^{*}$ cells grown in PYE. Biological

168 triplicate experiments are shown. Error bars represent $95 \%$ confidence interval. Inset

169 shows motility assays as measured by growth in $0.3 \%$ PYE agar.

170 B. Representative phase-contrast microscopy images of $w t, \Delta / o n$, and $\Delta / o n$ clp $X^{*}$ cells

171 grown in PYE during exponential phase. Quantification of cell length and stalk length for

172 three biological replicates of $n=100$ cells.

173 C. Serial dilution assays comparing colony formation of strains in PYE and PYE supplemented with mitomycin C. Spots are plated 10 -fold dilutions of exponentially

175 growing cells from left to right. 
176 D. Flow cytometry profiles showing chromosome content of indicated strains after three-

177 hour treatment with rifampicin. Cells were stained with SYTOX Green to measure DNA

178 content. The fluorescent intensities corresponding to one chromosome (1) and two

179 chromosomes (2) are indicated. Experiment was performed two times. Representative

180 data from one of the biological replicates is shown here.

181 E. Venn diagram summarizing the number of differentially expressed genes with an

182 FDR cutoff < 0.01 from RNA seq performed with stationary phase cells. Venn diagram

183 created by BioVenn (Hulsen et al., 2008).

184

185

See also Figure S1.

186

\section{ClpX* restores degradation of Lon substrates in vivo}

188 Because cells lacking Lon would accumulate normally degraded substrates, we hypothesized that the rescue we observed in the $\Delta / o n c l p X^{*}$ strain was likely due to lower levels of Lon substrates, such as DnaA, CcrM, and SciP (Figure 2A) (Gora et al.,

191 2013; Jonas et al., 2013; Wright et al., 1996). As expected, in unsynchronized cells,

192 DnaA and CcrM levels are higher in the $\Delta$ lon strain than in wildtype (Figure 2B).

193 Similarly, SciP levels are higher in $\Delta$ lon swarmer cells in comparison to wildtype (Figure

194 2D). Interestingly, levels of DnaA and SciP are restored to wildtype levels in the $\Delta / o n$

$195 c / p X^{*}$ strain; however, CcrM remained at higher levels (Figure 2B). The reduced levels

196 of DnaA likely explain the rescue of replication defects and enrichment analysis of the

197 RNA-seq results shows that the SciP-controlled regulon and cell-cycle gene expression

198 are largely restored in the $\Delta$ lon clpX* strain (Figure S2). We reasoned that expression

199 of $c / p X^{*}$ rescues normal growth and critical stress responses in a $\Delta$ lon strain through

200 restoring wildtype levels of key proteins.

202 Next, we explored the mechanism by which a mutation in ClpX is able to restore steady 203 state levels of DnaA and SciP. Because DnaA and SciP are controlled by proteolysis, 204 we suspected that protein turnover may be affected. As expected, DnaA is largely 205 stabilized in $\Delta$ lon strains (Figure 2C; Jonas, et al. 2013); however, we found that DnaA 206 degradation was restored in the $\Delta$ /on $c / p X^{*}$ strain, with a half-life similar to that of 207 wildtype (Figure 2D). SciP protein dynamics and cell-cycle phase specific levels were 208 also restored in $\Delta$ lon clpX* cells (Figure 2D). By contrast, CcrM degradation was still 
209 solely dependent on Lon, even when clpX* was present, in both unsynchronized cells

210 (Figure 2C) and during cell-cycle progression (Figure S3A).

212 We considered that changes in protein turnover could be either due to a gain-of-function

213 in the $c / p X^{*}$ mutant or by indirect cellular effects associated with a loss-of-function in

$214 c / p X$. To test this, we created a merodiploid $\Delta$ lon strain expressing a second copy of

$215 c / p X$ or $c / p X^{*}$ under control of the native promoter along with a normal chromosomal

216 copy of $c / p X$. We reasoned that if the effect was direct, then expression of $c / p X^{*}$ would

217 be sufficient to restore DnaA degradation. If the effect was indirect, then DnaA would

218 remain stable. Consistent with a gain-of-function phenotype for the clp $X^{*}$ allele, we

219 found that DnaA turnover increased upon expression of $c / p X^{*}$ but not wildtype $c / p X$

220 (Figure 2E). Similarly, the phenotypic rescue we observed in the $\Delta$ lon clpX* strain was

221 also due to a gain-of-function activity as the merodiploid strain expressing clp $X^{*}$

222 suppressed $\Delta$ lon defects, such as growth, motility, and genotoxic stress tolerance

223 (Figure S3B-D). Because CIpXP is a AAA+ protease, we hypothesized that ClpX*P (the

224 protease complex of the variant $\mathrm{ClpX}^{*}$ with wildtype ClpP) was now able to recognize

225 and degrade substrates normally degraded by Lon. 
Figure 2

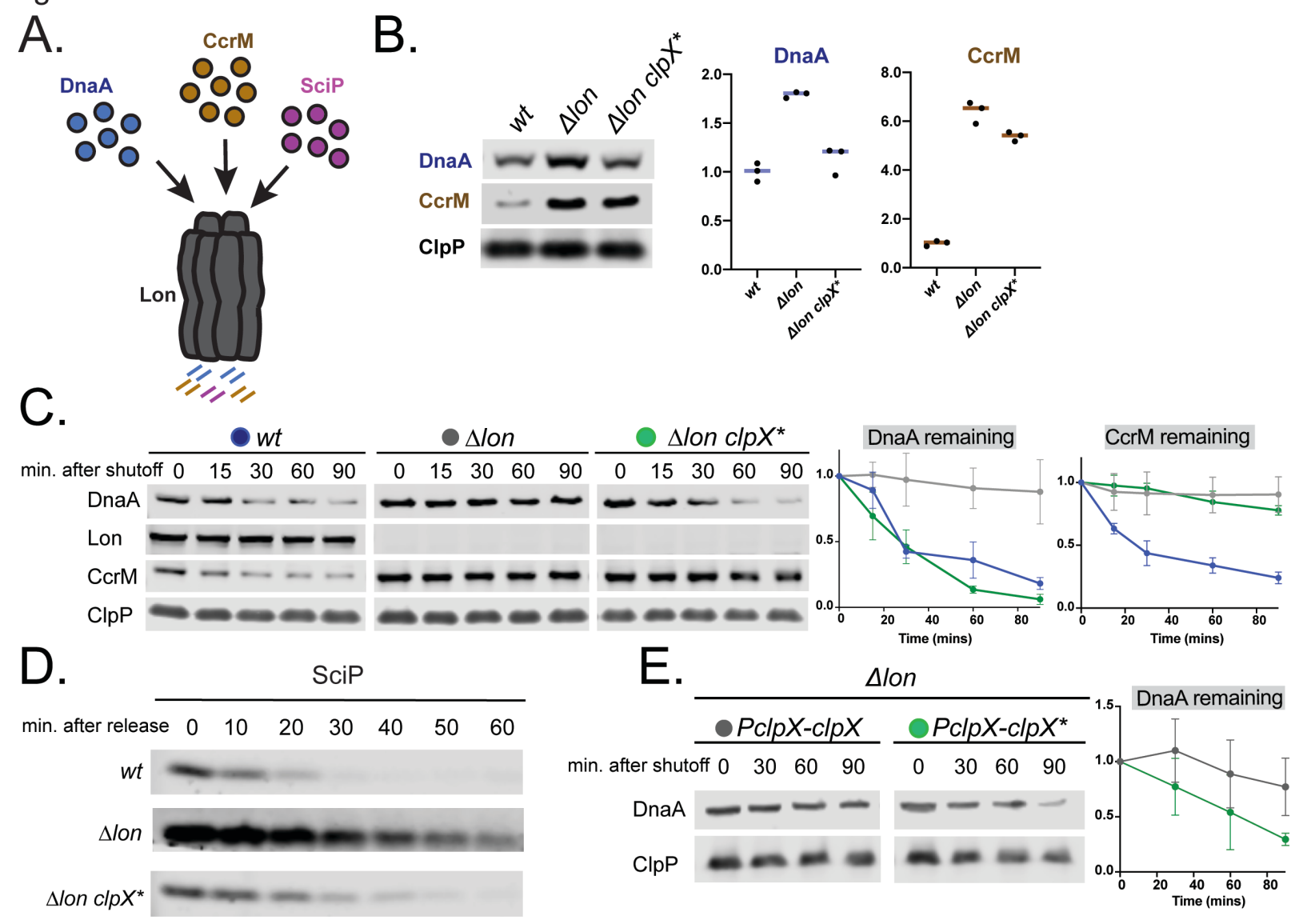

Figure 2: clp $X^{*}$ mutant restores levels of Lon substrates through degradation

228 A. Lon degrades DnaA, SciP, and CcrM in C. crescentus (Wright et al., 1996; Gora et

229 al., 2013; Jonas et al., 2013).

230 B. Western blot showing DnaA and CcrM levels in wt, $\Delta /$ on, and $\Delta / o n$ clpX* cells.

231 Lysates from an equal number of exponential phase cells were probed with anti-DnaA

232 or anti-CcrM antibody. ClpP was used as a loading controls. A representative image

233 and quantifications of triplicate experiments are shown.

234 C. Antibiotic shutoff assays to monitor DnaA and CcrM stabilities in wt, $\Delta / o n$, and $\Delta / o n$ clp $X^{*}$ cells. Chloramphenicol was added to stop synthesis and lysates from samples at the indicated time points were used for western blot analysis. Quantifications of triplicate experiments with substrate shown relative to ClpP control levels are shown to the right. Error bars represent SD. D. Western blot showing SciP levels in synchronized populations of wt, $\Delta / o n$, and $\Delta / o n$ $c / p X^{*}$ cells. Swarmer cells were isolated using a density gradient and an equal number of cells were released into fresh PYE medium. Samples were withdrawn at the indicated time points and probed with anti-SciP. $\mathrm{E}$. DnaA stability is measured in $\Delta /$ on strains expressing an extra copy of wildtype clpX or $c / p X^{*}$. Experiment was performed four times. Representative data from one of the

246 Error bars represent SD. 
See also Figure S2 and S3.

\section{ClpX*P directly degrades Lon substrates in vitro}

251 As a direct test of our hypothesis, we purified the ClpX* variant and reconstituted

252 degradation in vitro. Upon initial characterization of purified $\mathrm{ClpX}^{*}$, we found that it 253 formed an active ATPase, but hydrolyzed ATP three times faster than wildtype (Figure 254 S4A). Consistent with the fact that Lon is the principal protease for DnaA degradation 255 (Jonas et al., 2013; Liu et al., 2016), we found that wildtype ClpXP poorly degraded 256 DnaA in vitro (Figure $3 \mathrm{~A})$. However, purified CIpX*P could degrade DnaA four-fold 257 faster than CIpXP (Figure 3A). Similarly, SciP was degraded three-fold faster by CIpX*P 258 in comparison to CIpXP (Figure 3A). Finally, as predicted from our in vivo results, CcrM 259 was not degraded by either CIpX*P or CIpXP (Figure 3A, Figure S4B), but was well 260 degraded by Lon (Figure S4B). Thus, ClpX* appears to have an expanded substrate profile which includes some Lon substrates, but others, such as CcrM, remain exclusive 262 to Lon. Lon recognizes exposed hydrophobic amino acids (Gur and Sauer, 2008) and is best known as a quality control protease that eliminates mis/unfolded proteins (Goff et al., 1984). Given its altered specificity, we considered if ClpX* could now recognize these substrates. Casein is commonly used as an in vitro model substrate for mis/unfolded proteins. Lon robustly degrades fluorescently labeled casein, whereas wildtype CIpXP poorly degrades this substrate (Figure 3B). Intriguingly, CIpX ${ }^{*} \mathrm{P}$ degrades FITC-casein more than twice as fast as CIpXP (Figure 3B, Figure S4C). Similar to casein, CIpX*P

271 degrades another unfolded Lon substrate, a chemically denatured domain of titin tagged with the hydrophobic sequence $\beta 20$, better than wildtype CIpXP (Figure S4D).

Because cells lacking Lon are sensitive to proteotoxic stresses that create misfolded proteins and because $\mathrm{ClpX}^{*} \mathrm{P}$ is better at degrading mis/unfolded substrates than wildtype CIpXP, we wondered if the $\Delta$ lon clpX* strain would be more resistant to stressors that induce cellular protein misfolding. Indeed, the presence of the clp $X^{*}$ allele 
(Figure 3B). We conclude that $\mathrm{ClpX}^{*}$ has an altered substrate preference, that includes

281 Lon protease.

Figure 3
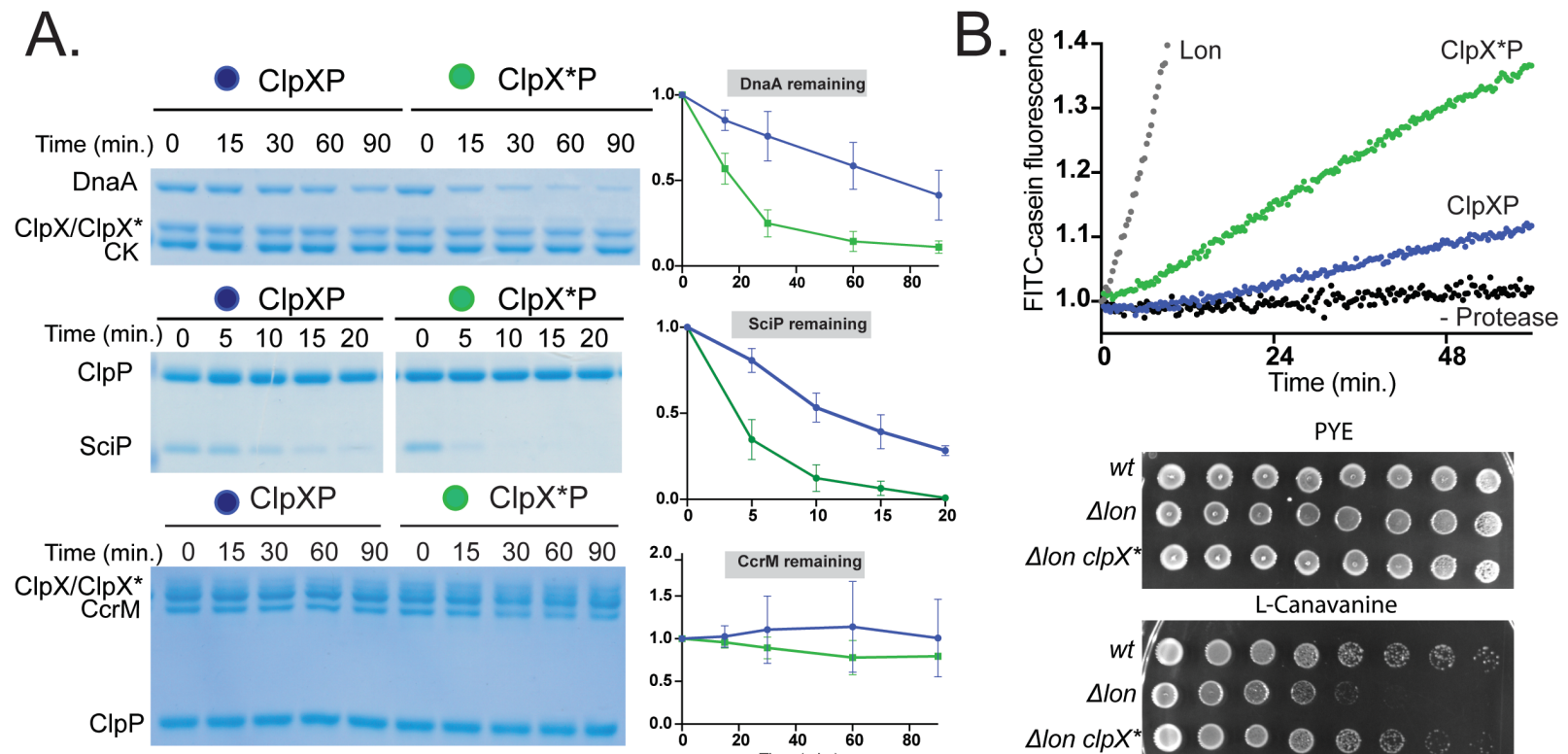

Figure 3: CIpX*P degrades some Lon substrates faster than ClpXP in vitro. A. In vitro degradation of DnaA, SciP, or CcrM. Assays were performed with $0.1 \mu \mathrm{M}$ $\mathrm{ClpX}_{6}$ or $\mathrm{ClpX}_{6}{ }^{*}$, and $0.2 \mu \mathrm{M} \mathrm{ClpP}_{14}$. Substrate concentrations were $1 \mu \mathrm{M}$ DnaA, $5 \mu \mathrm{M}$ SciP, $0.5 \mu \mathrm{M}$ CcrM. Quantification of triplicate experiments shown. Error bars represent SD.

B. In vitro fluorescence degradation assay of FITC-casein in the presence of CIpXP or ClpX*P or Lon. Degradation assays were performed with $10 \mu \mathrm{g} / \mathrm{mL}$ FITC-Casein, $0.1 \mu \mathrm{M}$ $\mathrm{ClpX}_{6}$ or $\mathrm{ClpX}_{6}{ }^{*}$ and $0.2 \mu \mathrm{M}$ ClpP or $0.1 \mu \mathrm{M}$ Lon 6 . Serial dilution assays comparing colony formation of strains in PYE and PYE supplemented with L-canavanine. Spots are plated 10-fold dilutions of exponentially growing cells from left to right.

See also Figure S4.

\section{ClpX*P is deficient in degradation of native CIpXP substrates}

Mutations in ClpX that improve recognition of some substrates can have negative consequences on others (Farrell et al., 2007). GFP-ssrA is a well-characterized CIpXP substrate that is directly bound by the pore loops of ClpX (Fei et al., 2020; Gottesman et al., 1998). We purified GFP fused to the Caulobacter ssrA tag (Chien, et al. 2007), determined degradation rates by $\mathrm{ClpX}{ }^{*} \mathrm{P}$ and $\mathrm{ClpXP}$, and fit these rates to the Michaelis-Menten equation. We found that ClpX*P degrades GFP-ssrA more poorly 
303 than wildtype CIpXP, principally lowering the turnover rate (Figure 4A, Figure S5A),

304 suggesting that either ClpX* fails to bind the ssrA tag as readily or is unable to

305 translocate the reporter protein as well as ClpX. This effect was not due to the GFP as

306 an ssrA-tagged unfolded titin domain was also degraded more slowly by CIpX*P (Figure

307 S5B). Given that the $\beta 20$-tagged version of this same construct was degraded more

308 rapidly, we conclude that the altered specificity of ClpX* must rely on the recognition tag

309 identity, rather than on changes in substrate translocation

311 CtrA is a master regulator in Caulobacter that inhibits replication initiation and regulates

312 the transcription of many cell-cycle genes (Laub et al., 2002; Wortinger et al., 2000). In

313 vivo, CtrA is degraded during the G1-S transition by ClpXP through the use of an

314 adaptor hierarchy (Domian et al., 1999; Jenal and Fuchs, 1998; Joshi et al., 2015; Quon

315 et al., 1996); however, in vitro CtrA can also be directly recognized by CIpXP as its C-

316 terminus resembles the ssrA tag (Chien et al., 2007). Consistent with a reduced ability

317 to recognize ClpXP substrates, ClpX*P degrades isolated CtrA more poorly than

318 wildtype (Figure 4B) and also shows reduced degradation of a CtrA-derived GFP

319 reporter (Smith et al., 2014) in the presence of the full adaptor hierarchy (Figure S5C).

320 We conclude that $\mathrm{ClpX} P$ overall has a diminished ability to recognize and degrade

321 native substrates normally degraded by CIpXP.

\section{Deficiencies of ClpX* revealed when Lon is present}

324 The prior in vitro data suggests that rather than just expanding specificity, this mutation

325 has allowed $\mathrm{ClpX}^{*} \mathrm{P}$ to degrade Lon-dependent substrates better but at the cost of

326 degrading CIpXP-dependent ones. If this is true, while $c / p X^{*}$ is beneficial in a $\Delta l o n$

327 background, we reasoned that it may be detrimental to lon $^{+}$cells.

329 To test this hypothesis, we generated a strain with $c / p X^{*}$ as its sole copy. We first

330 examined ssrA tag turnover in this strain by using an ssrA derivative that relies on the

331 SspB adaptor for degradation (eGFP-ssrA(DAS)) (Chowdhury et al., 2010) so that

332 degradation would be sufficiently slowed to be visualized by western blotting.

333 Consistent with our in vitro experiments, we observed stabilization and dramatically 
334 higher levels of eGFP-ssrA (DAS) in the clpX* strain in comparison to wildtype (Figure

$3354 \mathrm{C})$. We observed reduced CtrA degradation in the clpX* strain, consistent with our in

336 vitro observations (Figure 4C). Finally, DnaA degradation is accelerated in the $c / p X^{*}$

337 strains compared to wildtype - consistent with our findings that both Lon and ClpX*P

338 are able to degrade DnaA (Figures 4C).

340 We assayed the overall physiological consequence of ClpX* using competitive fitness in

341 mixed cultures. We grew cultures of wildtype $c / p X$ or the $c / p X^{*}$ strain together with

342 wildtype strains constitutively expressing the Venus fluorescent protein (Persat et al.,

343 2014). We found that while nonfluorescent wildtype cells could slightly outcompete the

344 constitutive Venus expressing cells, the $c / p X^{*}$ strain showed a substantial competitive

345 disadvantage (Figure 4D). We also tested for stress tolerances and found that $c / p X^{*}$

346 strains fail to survive genotoxic stress as robustly as wildtype (Figure 4E). Our

347 conclusion is that reduced fitness of the wildtype $c / p X^{*}$ strain is likely due to reduced

348 degradation of normal ClpXP substrates (such as CtrA) and prolific degradation of

349 normal Lon substrates (such as DnaA) that together reduce overall fitness. Collectively,

350 this supports our understanding that AAA+ proteases have been optimized for specific

351 priorities in a cell - expansion of their substrate profile, as seen with clp $X^{*}$, can

352 compensate for loss of other proteases, but at the cost of their ability to recognize their

353 normal substrates. 
Figure 4

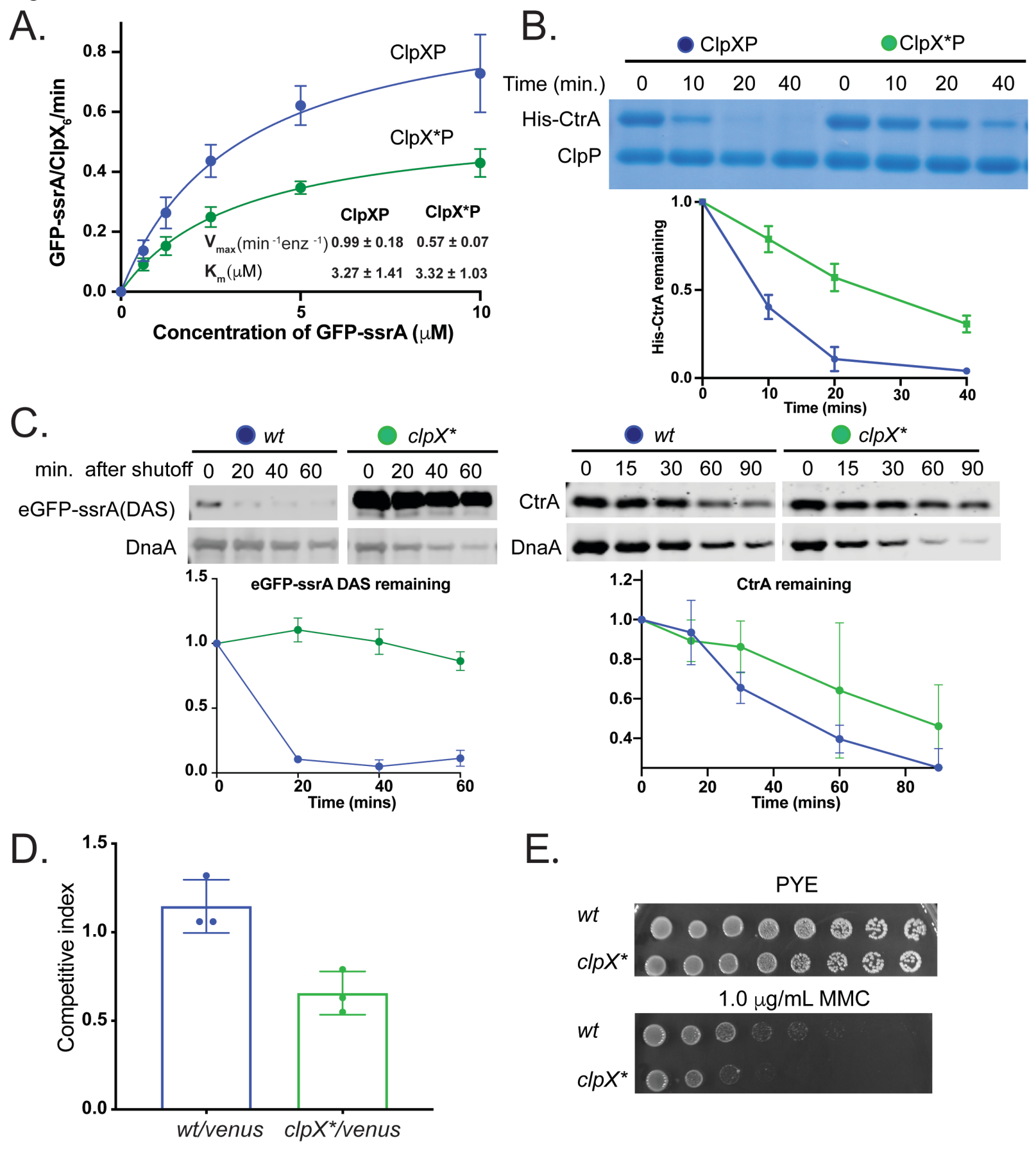

Figure 4: $\mathrm{ClpX}^{*}$ mutant is deficient in degradation of native CIpXP substrates concentration by $\mathrm{ClpXP}$ and $\mathrm{ClpX}{ }^{*} \mathrm{P}$. Inset displays kinetic parameters. Assays were performed with $0.1 \mu \mathrm{M}$ ClpX or ClpX* hexamer, $0.2 \mu \mathrm{M}$ ClpP, ATP regeneration system, and the indicated concentrations of GFP-ssrA. Data was fitted to Michaelis-Menten 
B. In vitro degradation assay of His-CtrA in the presence of ClpXP or ClpX*P. Degradation assays were performed with $3 \mu \mathrm{M}$ His-CtrA, $0.1 \mu \mathrm{M}$ ClpX or ClpX* hexamer, $0.2 \mu \mathrm{M} \mathrm{ClpP}$, and ATP regeneration system. Quantification of duplicate experiments is shown below. Error bars represent SD. and $c / p X^{*}$ cells. The plasmid encoded M2FLAG-eGFP-ssrA (DAS) construct was induced with the addition of $0.2 \%$ xylose. Chloramphenicol was used to inhibit protein synthesis. Samples were withdrawn at the indicated time points and quenched in SDS lysis buffer. Lysate from an equal number of cells was used for western blot analysis and probed with anti-M2, or anti-CtrA, and anti-DnaA antibody. Quantification of triplicate experiments is shown below. Error bars represent SD. D. Competition assay with wildtype cells harboring xylX::Plac-venus (constitutive venus expression) and nonfluorescent wt or $c / p X^{*}$ strains. exponential phase cells were mixed 1:1, diluted, and allowed to outgrow for 12 doublings. . Quantification of triplicate experiments is shown below. Error bars represent SD. PYE supplemented with MMC. Spots are plated 10-fold dilutions of exponentially

\section{Limiting ATP alters wildtype CIpXP substrate specificity}

To understand how this mutation in ClpX leads to enhanced degradation of certain substrates, we further characterized the ATPase activity of Clp $X^{*}$. While ClpX* hydrolyzes ATP three-fold faster than wildtype ClpX at saturating ATP concentrations

(Figure S4A), Michaelis-Menten experiments showed that the $K_{M}$ increases by 5-6 fold and the catalytic efficiency for ATP hydrolysis decreases by approximately 2 fold,

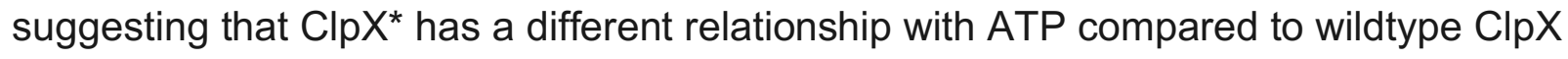
(Figure 5A). As seen previously, degradation of GFP-ssrA was precipitously lost below a minimum threshold of ATP (Martin et al., 2008), with the ClpX*P threshold being

391 higher than that of wildtype, consistent with the higher $\mathrm{K}_{\mathrm{M}}$ for ATP described above

392 (Figure S6A). For ClpX*P, casein degradation reduced monotonically as ATP 393 concentration was lowered (Figure 5B). In striking contrast, we found that for wildtype

394 ClpXP, casein degradation was increased at intermediate nucleotide concentrations 395 compared to saturating ATP (Figure 5B). Kinetic analysis shows that at these lower 396 ATP concentrations ClpXP degrades casein with a lower $\mathrm{K}_{\mathrm{M}}$ and a similar $\mathrm{V}_{\max }$ 
$398 \mathrm{~K}_{\mathrm{M}}$ rate suggests that, at a minimum, casein must bind better to ClpX at these concentrations.

These results suggest that CIpX specificity depends on ATP concentration in an unexpected fashion, with lower levels of ATP facilitating ClpX recognition of some substrates. We reasoned that this could explain the altered specificity of the ClpX* mutant, as its increased $\mathrm{K}_{\mathrm{M}}$ for ATP may allow $\mathrm{ClpX}^{*}$ to resemble this low ATP state even under saturating ATP conditions (Figure 5A). Remarkably, this enhanced degradation at low ATP extended to other ClpX* substrates, such as DnaA and SciP (Figure 5D and S6B). We conclude that CIpX preference depends on ATP state, with its specificity expanded when not fully loaded with ATP.

This in vitro data suggests that CIpXP may play a role in better degrading some 411 substrates such as DnaA in vivo under ATP limiting conditions. To test this, we

412 monitored degradation of DnaA during starvation using a $\triangle$ lon $\triangle c / p A$ strain which should 413 eliminate any non-ClpX dependent degradation (Liu et al., 2016). Glucose starvation 414 has been shown to accelerate DnaA degradation (Gorbatyuk and Marczynski, 2005)

415 which we found true for wildtype cells as well (Figure 5E). Importantly, starving $\Delta / o n$ $416 \Delta c l p A$ also markedly increased DnaA degradation (Figure 5E) concurrent with a 417 reduction in ATP levels (Figure S6C).

We considered conformational changes in CIpX that might explain the altered specificity at low ATP. Using limited proteolysis to probe conformational differences we found that 421 ClpX at low ATP was more protease accessible than at high ATP (Figure 5F).

422 Consistent with the shared altered specificity, protease accessibility of ClpX*at high 423 ATP was more similar to wildtype ClpX at low ATP than high (Figure 5F). This suggests 424 that a more open structure of ClpX is present at low ATP and with ClpX* that allows it to 425 recognize some substrates more readily. We hypothesize that this low ATP-induced 426 conformational difference may reflect the observed changes in AAA+ structures seen in 427 recent cryo-EM studies and discuss their ramifications below. 
Figure 5

A.
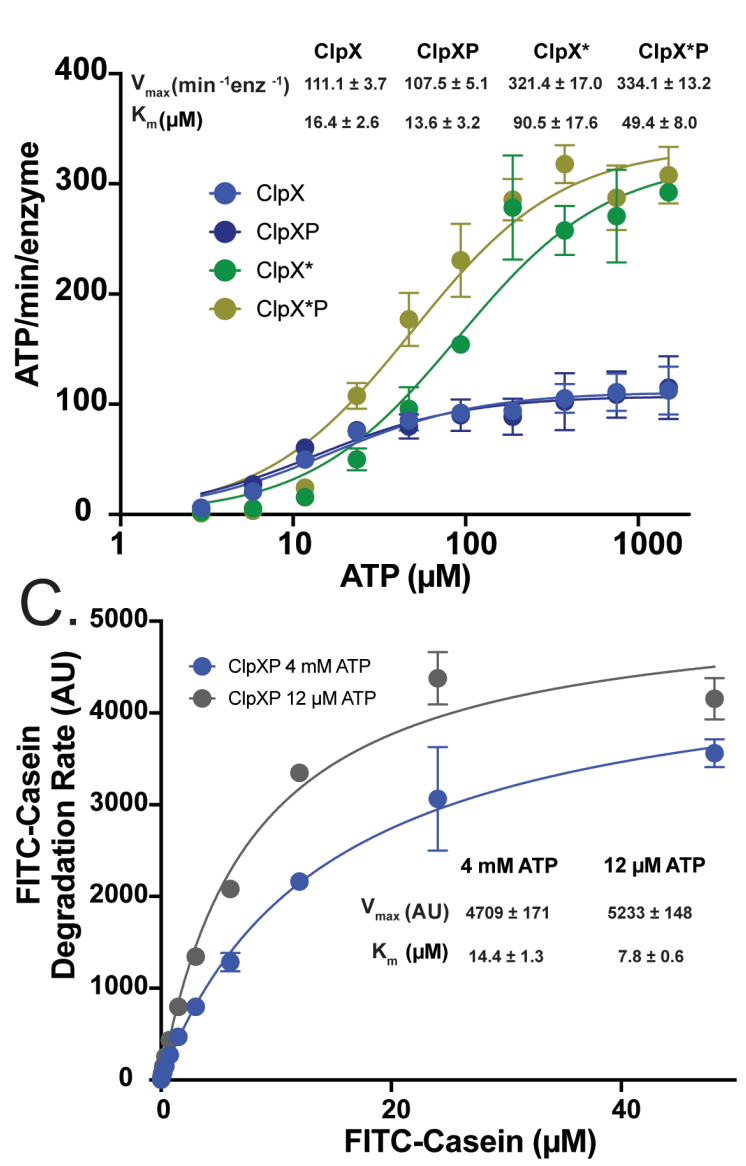

E.

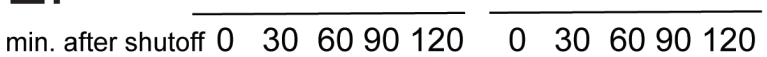

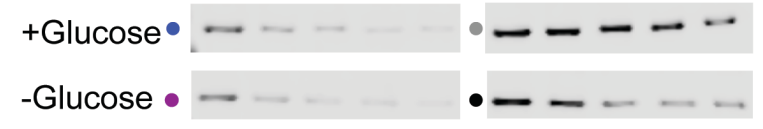

DnaA remaining
B.

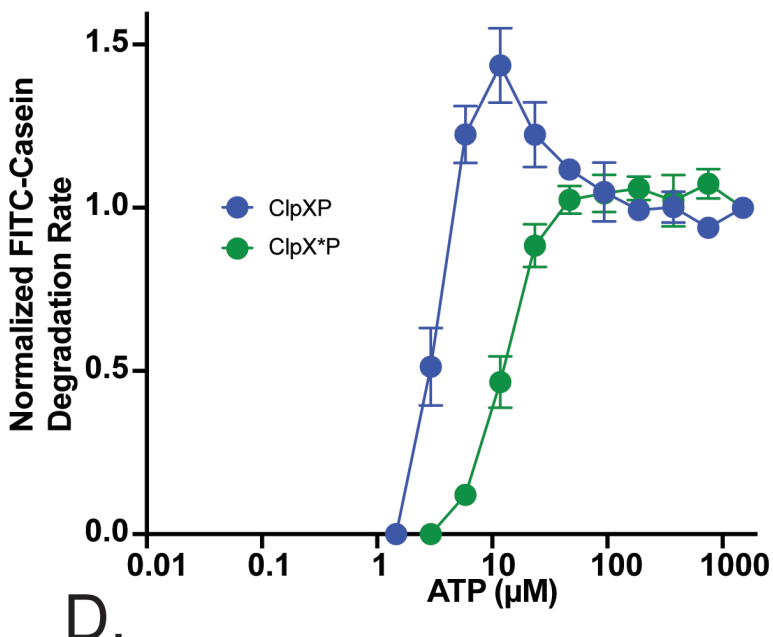

$\frac{12 \mu \mathrm{M} \text { ATP }}{03060901200306090120}$

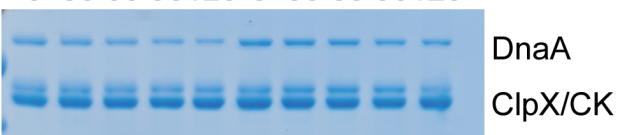

F.

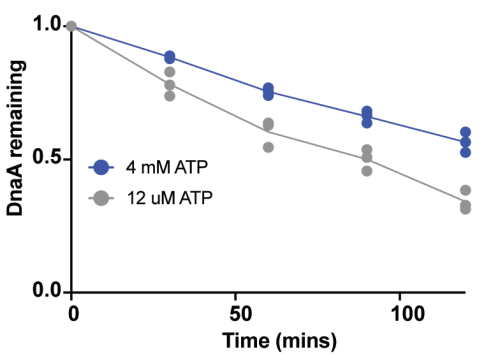

$\mathrm{ClpX} \quad \mathrm{ClpX} \mathrm{X}^{*}$ min. after $\frac{4 \text { mM ATP }}{05102040} \frac{12 \text { uM ATP }}{5102040} \frac{4 \text { mM ATP }}{05102040}$ chymotrypsin addition

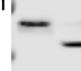

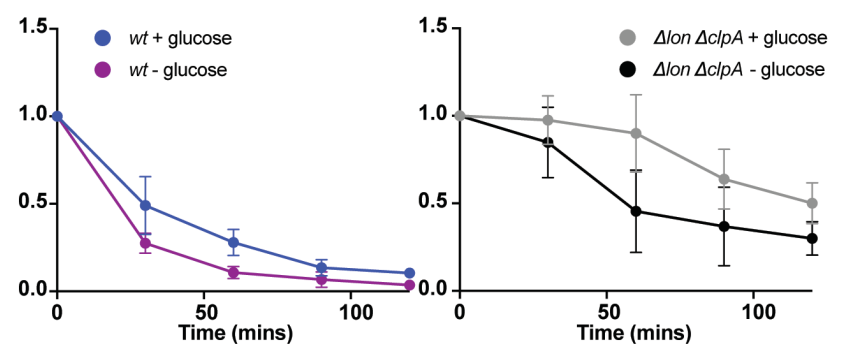

429 Figure 5: Limiting ATP alters wildtype ClpXP substrate specificity

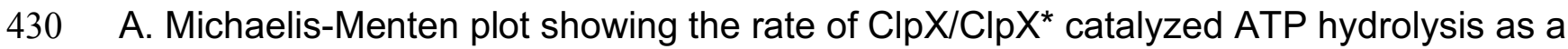
function of ATP concentration. Inset displays kinetic parameters. Assays were 
performed with $0.1 \mu \mathrm{M}$ CIpX or $\mathrm{ClpX}^{*}$ hexamer, in the presence and absence of $0.2 \mu \mathrm{M}$ CIpP. Data was fitted to Michaelis-Menten equation. Quantification of duplicate experiments.

B. FITC-Casein degradation by CIpXP or ClpX*P as a function of ATP concentration. Assays were performed with $0.1 \mu \mathrm{M}$ ClpX or ClpX* hexamer, $0.2 \mu \mathrm{M} \mathrm{ClpP,} \mathrm{ATP}$ regeneration system, and $50 \mu \mathrm{g} / \mathrm{mL}$ FITC-Casein. Quantification of duplicate experiments.

C. Michaelis-Menten plot showing the rate of degradation as a function of FITC-Casein concentration by CIpXP and CIpX*P under low and saturating ATP conditions. Inset displays kinetic parameters. Assays were performed with $0.1 \mu \mathrm{M} C l p X$ or $\mathrm{ClpX}^{*}$ hexamer, $0.2 \mu \mathrm{M}$ CIpP, ATP regeneration system. Data was fitted to Michaelis-Menten equation. Quantification of triplicate experiments D. In vitro degradation of DnaA by CIpXP under low and saturating ATP conditions. Assays were performed with $0.1 \mu \mathrm{M} \mathrm{ClpX}_{6}$ or $\mathrm{ClpX}_{6}{ }^{*}$, and $0.2 \mu \mathrm{M} \mathrm{ClpP} 14$ and $0.5 \mu \mathrm{M}$ DnaA. Quantification of triplicate experiments.

E. Antibiotic shutoff assays to monitor DnaA under ATP limiting conditions in wt and $\Delta l o n \Delta c / p A$ strains. Chloramphenicol was added to stop synthesis and lysates from samples at the indicated time points were used for western blot analysis. Quantifications of triplicate experiments shown to the right. Error bars represent SD.

F. Limited proteolysis assay to probe conformational states. ClpX was incubated with Chymotrypsin in Hepes buffer (20 mM Hepes pH 7.5, $100 \mathrm{mM} \mathrm{KCl,} 10 \mathrm{mM} \mathrm{MgCl}$, 10\% glycerol) at $12 \mu \mathrm{M}$ ATP and $4 \mathrm{mM}$ ATP in the presence of ClpP and ATP regeneration system at $25^{\circ} \mathrm{C}$ for the indicated time points. ClpX* was incubated with Chymotrypsin in Hepes buffer (20 mM Hepes pH 7.5, $100 \mathrm{mM} \mathrm{KCl,} 10 \mathrm{mM} \mathrm{MgCl}, 10 \%$ glycerol) at 4 mM ATP in the presence of CIpP and ATP regeneration system at $25^{\circ} \mathrm{C}$ for the indicated time points. ClpX was detected using anti-ClpX antibodies.

See also Figure S6.

\section{Discussion}

Because proteolysis is irreversible, degradation of substrates must be carefully monitored to avoid toxic consequences. In bacteria, different energy dependent proteases have distinct substrate preferences that allows them to collectively regulate proteome dynamics. However, how these energy-dependent proteases choose their target substrates from a pool of cellular proteins remains poorly understood. Certain determinants that dictate specificity include degrons, or degradation tags, such as the ssrA tag which marks proteins for degradation by CIpXP or CIpAP (Keiler, 2015; Keiler et al., 1996). Specificity can be augmented through adaptor proteins that help deliver substrates to proteases. For example, degradation of CtrA in Caulobacter is restricted to the ClpXP protease via a highly regulated series of adaptors (Joshi et al., 2015). 
473 In this work, we identified a mutant allele of $c / p X\left(c / p X^{*}\right)$ that compensates for the loss

474 of Lon through expanding CIpXP substrate specificity. The variant ClpX*P protease

475 complements the growth, motility, replication status, and morphology defects of a $\Delta /{ }^{\circ} n$

476 strain (Figure 1A-1C) by restoring levels and degradation of the Lon substrates DnaA

477 and SciP (Figure 2), which we confirm with biochemical experiments (Figure 3).

478 Interestingly, ClpX*P has an improved ability to degrade unfolded protein substrates, a feature that likely explains the increased proteotoxic tolerance of $\Delta / o n c l p X^{*}$ strains

480 (Figure 3). Finally, this clpX variant is deficient in proteolysis of normal ClpXP

481 substrates, such as CtrA and ssrA-tagged proteins, suggesting a shift in substrate

482 preference rather than just an expansion (Figure 4). Mechanistic enzymology revealed

483 that $\mathrm{ClpX}^{*}$ requires $\sim 5$-fold more ATP for saturation (Figure 5A), leading us to explore

484 the role of ATP levels in controlling specificity. Surprisingly, our in vitro studies found

485 that wildtype ClpXP is better at degrading unfolded proteins, DnaA, and SciP in ATP

486 limiting conditions, but worse at degrading ssrA-tagged proteins, similar to that seen

487 with $\mathrm{ClpX}{ }^{*} \mathrm{P}$ in saturating ATP (Figure 5B, 5D, S6B). We recapitulate this effect in vivo,

488 showing that in starvation conditions that deplete ATP, DnaA degradation by wildtype

489 ClpXP is accelerated (Figure 5E). Our interpretation is that the ClpX oligomer adopts

490 distinct conformations in the ATP-saturated and ATP-limited conditions, as suggested

491 by limited proteolysis (Figure 5F).

493 How does altered ATP loading alter substrate recognition? Our working model is that

494 ClpX samples a 'open' state and a 'closed' state (Figure 6). Substrates can be captured during the open-closed transition (as we propose for casein) or directly by the closed

496 state (as proposed for the ssrA tag and CIpX (Hersch et al., 2005; Fei et al., 2020).

497 Once a closed state engages a substrate, the unfoldase can processively translocate 498 the substrate to ClpP in the case of ClpXP (Figure 6). Recent cryo-EM structures reveal 499 shared features of substrate-bound AAA+ complexes, namely a shallow right-handed 500 ring with pore loops gripping the substrate and at least 4 ATP-bound protomers (Fei et 501 al., 2020; Gates et al., 2017; Lopez et al., 2020; Ripstein et al., 2020; Shin et al., 2020). 502 Consistent with our sampling model, substrate-free forms of these machines exhibit 
503 more open, often left-handed, spiral structures (Shin et al., 2020; Yokom et al., 2016).

504 Interestingly, the original crystallization of ClpX shows a left-handed open spiral crystal

505 packing (Kim and Kim, 2003), similar to these substrate-free cryo-EM structures

506 described for other AAA+ machines. We propose that mutations or limiting ATP can

507 shift the open/closed balance to the more open state, which allows ClpX to better

508 capture certain substrates, such as unfolded proteins, which may not have as strict

509 sequence requirements for recognition. However, degrons such as the ssrA tag which

510 bind selectively to the closed conformation (Fei et al., 2020; Hersch et al., 2005) would

511 be poorly recognized in these conditions.

513 Prior work has shown that limiting nucleotide leads to loss of substrate degradation by

514 ClpXP (Martin et al., 2008), as would make sense for an ATP-fueled unfoldase.

515 However, we note that these studies exclusively used ssrA-tagged substrates, which

516 requires an ATP-bound (and presumably closed) form of ClpX for recognition. Thus,

517 the equivalent effects we are seeing here for casein, DnaA, and SciP would not have

518 been previously observed and also highlights the importance of using a range of

519 substrates in understanding activity. The open-capture / closed-processive model

520 described here also has precedence in the conformational states and activity of Hsp104

521 (Gates et al., 2017; Lee et al., 2019; Ye et al., 2020; Yokom et al., 2016), where doping

522 in nonhydrolyzable ATP analogs results in enhanced unfolding activity (Doyle et al.,

523 2007). In our current work, we have directly shown how different states of ClpX, favored

524 by either limiting ATP or mutation, can result in meaningful biological consequences, for

525 example by compensating for the loss of the Lon protease. Overall, we favor the

526 interpretation that exclusive substrate profiles for each AAA+ system are not hard-wired

527 but can be altered not only by mutations but also through modulation of ATP hydrolysis,

528 demonstrating a plasticity in these machines that yield flexibility in maintaining cellular

529 proteostasis. 
Figure 6
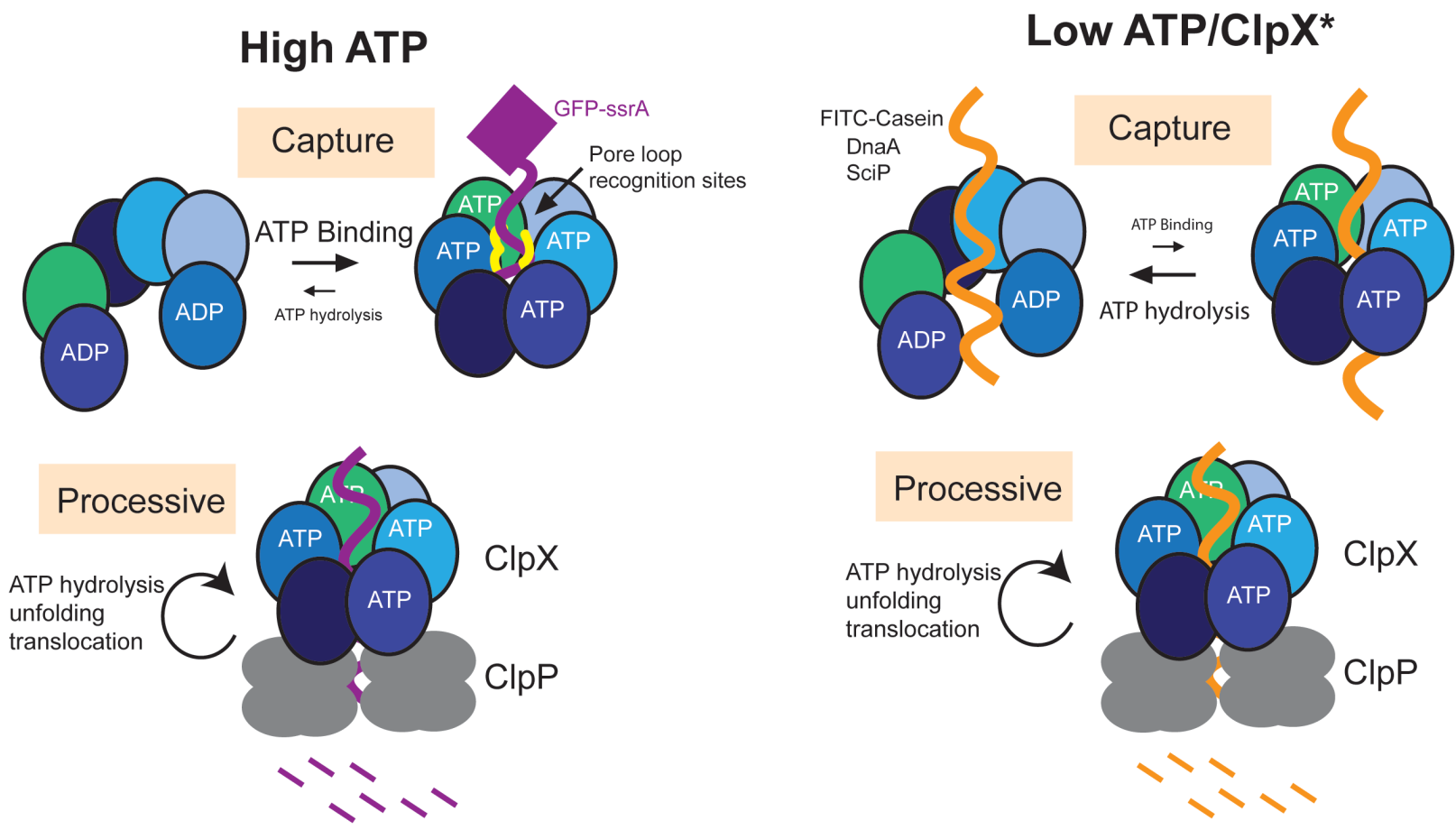

531 Figure 6: Model of ATP-dependent changes in CIpXP substrate specificity ClpX exists in an equilibrium between an open and closed conformation. Under high ATP conditions, the closed conformation is favored, allowing ClpX to recognize and processively degrade certain substrates, such as GFP-ssrA, which makes sequencespecific contacts with pore loops of CIpX in a specific conformation (show in yellow). GFP-ssrA can then be unfolded and translocated to ClpP for irreversible degradation. Under low ATP conditions or in the presence of ClpX*, the balance shifts towards the open state, allowing CIpX to capture non-traditional classes of substrates, such as casein. ClpX can then switch to the closed state allowing processive degradation, unfolding, and translocation of captured substrate.

\section{Materials and Methods}

\section{Bacterial strains and growth conditions}

544 Bacterial strains and plasmids used in this study are listed in supplementary file 2.

545 Caulobacter crescentus strains were grown in PYE medium (2g/L peptone, $1 \mathrm{~g} / \mathrm{L}$ yeast

546 extract, $1 \mathrm{mM} \mathrm{MgSO}_{4}$, and $0.5 \mathrm{mM} \mathrm{CaCl}_{2}$ ) at $30^{\circ} \mathrm{C}$. To generate strains, antibiotics were 547 added to plates at the following concentrations: kanamycin $(25 \mu \mathrm{g} / \mathrm{ml})$, spectinomycin

$548(100 \mu \mathrm{g} / \mathrm{ml})$, and oxytetracycline $(2 \mu \mathrm{g} / \mathrm{ml})$. After initial selection steps for strain 
crescentus motility assays, PYE with $0.3 \%$ agar was used and a single colony was stabbed into the agar using a sterile tip and left to incubate at $30^{\circ} \mathrm{C}$ for 2 to 3 days.

Escherichia coli strains were grown in LB $(10 \mathrm{~g} / \mathrm{L} \mathrm{NaCl}, 10 \mathrm{~g} / \mathrm{L}$ tryptone, $5 \mathrm{~g} / \mathrm{L}$ yeast extract) and supplemented with antibiotics at the following concentrations: ampicillin (100 ug/ml), oxytetracycline $(15 \mu \mathrm{g} / \mathrm{ml})$, spectinomycin $(50 \mu \mathrm{g} / \mathrm{ml})$, kanamycin (50 $\mu \mathrm{g} / \mathrm{ml})$, gentamycin $(20 \mu \mathrm{g} / \mathrm{ml})$. For solid medium, 1.5\% agar was used regardless of bacterial species. For all strains, optical density was measured at $600 \mathrm{~nm}$.

For induction purposes, the 477 plasmids were induced with xylose $(0.2 \%)$ and repressed with glucose (0.2\%). For protein expression, $0.4 \mathrm{M}$ IPTG was used for

561 induction.

562

\section{Cloning and strain constructions}

564 All Caulobacter strains were derived from CPC176, an isolate of NA1000. To generate 565 the $\Delta$ lon clpX* strain, a two-step recombination protocol with a sucrose counter 566 selection was utilized (Skerker et al., 2005). The vector pNPTS138 was digested with

567 HindIII and EcoRI. A PCR product of the $c l p X^{*}$ sequence was amplified from the original

568 motility suppressor and Gibson assembly was then used to generate pNPTS138_clpX*.

569 Following transformation with pNPTS138_clpX* into NA1000 or $\Delta$ lon, primary selection

570 was on PYE supplemented with kanamycin. Primary colonies were grown overnight

571 without selection and overnight cultures were played on PYE agar supplemented with

$5723 \% \mathrm{w} / \mathrm{v}$ sucrose. To validate allelic swap, strains were tested for sensitivity to

573 kanamycin. $\Delta$ lon $c / p X^{*}$ was also screened by motility and sequencing of the clpX locus

574 validated candidate clones.

$576 C / p X$ or $c / p X^{*}$ merodiploid strains were generated by Gibson assembly of PCR product 577 and double digested plasmids of pMCS-2. The plasmids were then electroporated into $578 \Delta / o n$ and selected onto kanamycin plates. Strains were validated by anti-ClpX westerns. 
580 eGFP-ssrA(DAS) induction strains were generated by electroporating eGFP-ssrA(DAS)

581477 plasmid into wildtype and wildtype $c / p X^{*}$ and selecting on spectinomycin. Strains

582 were validated by anti-M2 westerns.

583

\section{Motility Suppressor screen}

585 Transposon libraries were generated for $\Delta$ lon. Two-liter PYE cultures were grown to mid 586 exponential phase, pelleted, and washed with $10 \%$ glycerol. Competent cells were electroporated with Ez-Tn5 <Kan-2> transposome (Lucigen, Madison, WI). Cells recovered for 90 minutes at $30{ }^{\circ} \mathrm{C}$ and then played on PYE plates supplemented with kanamycin. Libraries were grown for 7 days. Colonies were then scraped from the surface, combined, and resuspended to form a homogenous solution of PYE $+20 \%$

591 glycerol.

592

593 The Tn library was thawed out and diluted into a flask containing two-liter $0.3 \%$ agar.

594 The cell agar mixture was plated and grown at $30^{\circ} \mathrm{C}$ for 3 to 5 days. Candidates that

595 appeared motile were validated by innoculating single colonies into motility agar on the

596 same plate as NA1000 as a positive control and $\Delta / o n$ as a negative control and

597 incubating plates for $2-3$ days at $30^{\circ} \mathrm{C}$.

\section{Whole genome sequencing}

600 Genomic DNA was extracted using the MasterPure Complete DNA and RNA

601 purification kit (Epicenter Biotechnologies, Madison, WI). A Qubit Fluorometer

602 (ThermoFisher Scientific, Waltham, MA) was utilized to assess DNA concentration.

603 Illumina libraries were generated from the extracted genomic DNA using the NexteraXT

604 (Illumina, San Diego, CA) protocol. Libraries were multiplexed and sequenced at the 605 University of Massachusetts Amherst Genomics Core Facility on the NextSeq 500 606 (Illumina). Single nucleotide polymorphisms (SNPs) were detected using breseq

607 (Deatherage and Barrick, 2014).

608 


\section{RNA sequencing}

612 RNA was extracted from stationary phase cells. Libraries were generated from the

613 extracted RNA using the NEB Next RNA Library Prep kits (NEB, Ipswich, MA). Libraries

614 were sequenced at the University of Massachusetts Genomics Core Facility on the

615 NextSeq 500 with single end 75 base reads. Reads were mapped to the Caulobacter

616 genome using BWA (Li and Durbin, 2009) and sorted with Samtools (Li et al., 2009).To

617 obtain the number of reads per gene, bedtools map (Quinlan and Hall, 2010) was used.

618 Rstudio and EdgeR was used to identify differentially expressed genes (R Core Team,

619 2019; Robinson et al., 2009).

621 Raw counts were normalized using the counts-per-million (CPM) method, and the

622 edgeR package in $\mathrm{R}$ was used to perform differential gene expression analysis, KEGG

623 pathway analysis, and FRY (Wu et al., 2010) gene set analysis. We filtered out any

624 gene that had less than 30 normalized counts across 3 or more samples. To test for

625 gene expression differences and identify differentially expressed genes across

626 experimental groups, we used the quasi-likelihood F-test. The over-representation

627 analysis for KEGG pathways done using the kegga function. FRY gene set analysis

628 done using the fry function. The CcrM, DnaA, and SciP regulons were obtained from

629 (Gonzalez et al., 2014; Hottes et al., 2005; Tan et al., 2010).

$631 \quad$ Plating viability and drug sensitivity

632 All Caulobacter strains were grown overnight in liquid PYE media. After overnight

633 growth, cells were back diluted to OD600 0.1 and outgrown to mid-exponential phase

634 before being normalized to $\mathrm{OD}_{600} 0.1$ and 10-fold serially diluted on to media. For

635 experiments using mitomycin C (Sigma, St. Louis, MO) and L-canavanine (Sigma),

636 drugs were prepared at a stock concentration of $0.4 \mu \mathrm{g} / \mathrm{ml}$ mitomycin C, and $100 \mathrm{mg} / \mathrm{ml}$

637 L-canavanine and filter sterilized. PYE agar was cooled before the drugs were added

638 and plates were left to air dry prior to serial dilution plating. All plates were incubated at

$63930{ }^{\circ} \mathrm{C}$ for $2-3$ days and imaged with a gel doc. 


\section{$642 \quad$ In vivo assays}

643 The stability of proteins in vivo was determined by inhibiting protein synthesis upon

644 addition of $30 \mu \mathrm{g} / \mathrm{ml}$ chloramphenicol to cells in exponential phase. At each time point,

$6451 \mathrm{ml}$ of culture was removed and centrifuged at 15,000 rpm for 2 minutes. The supernatant was removed and pellets were flash frozen in liquid nitrogen. Pellets were

647 thawed, resuspended in 2x SDS dye, and normalized to the OD 600 of the lowest sample.

648 Samples were boiled for 10 minutes and centrifuged for 10 minutes at 15,000 rpm.

649 Extracts were run on $10 \%$ Bis-Tris gels for 1 hour at room temperature at 150 V. Gels 650 were then transferred to nitrocellulose membranes for 1 hour at room temperature at 651 20V. Membranes were blocked with 3\% milk in Tris-based saline with 0.05\% Tween-20 652 (TBST) for 1 hour. Membranes were probed with primary antibody in 3\% milk in TBST 653 at $4^{\circ} \mathrm{C}$ overnight with following dilution factors: 1:5000 dilution of DnaA, 1:5000 SciP, 654 1:5000 CcrM, 1:5000 ClpP, 1:2500 Lon, and 1:2000 M2. Membranes were washed with 655 1x TBST for 5 minutes three times and then probed with Licor secondary antibody with $6561: 10,000$ dilution in 1x TBST at room temperature for 1 hour. The protein was visualized 657 using Licor Odyssey CLx. Bands were quantified using imageJ and degradation rates 658 were plotted using Prism.

660 To synchronize Caulobacter strains, cells were grown to an OD600 of 0.3-0.5 in PYE 661 and swarmer cells were isolated using Percoll density centrifugation. Cells were 662 released into PYE.

663 Flow cytometry was used to measure DNA content in rifampicin-treated cells as 664 previously described (Chen et al., 2009). Cells were rifampicin treated for three hours 665 prior to fixing in $70 \%$ ethanol.

\section{Carbon Starvation}

667 Cells were grown in M2G media (6.1 $\mathrm{mM} \mathrm{Na}_{2} \mathrm{HPO}_{4}, 3.9 \mathrm{mM} \mathrm{KH}_{2} \mathrm{PO}_{4}, 9.3 \mathrm{mM} \mathrm{NH}_{4} \mathrm{Cl}$, $6680.5 \mathrm{mM} \mathrm{MgSO}_{4}, 0.5 \mathrm{mM} \mathrm{CaCl}^{2}, 10 \mu \mathrm{M} \mathrm{FeSO}_{4}$ (EDTA chelate), $0.2 \%$ glucose). Cells 669 were then washed twice in cold M2G media (+glucose samples) or in M2 media (670 glucose samples) and resuspended in pre-warmed M2G or M2 media. 
671 For bulk measurements of intracellular ATP, the Bactiter-Glo microbial cell viability assay kit (Promega) was used. Cells were mixed with an equal volume of BacTiter-Glo reagent in 384-well microtiter plates and incubated for five minutes before luminescence

674 was measured.

\section{Growth competition assay}

676 Overnight cultures of CPC798 were mixed with either NA1000 or $c / p X^{*}$ at a 1:1 ratio.

677 Mixed strains were diluted into fresh PYE and allowed to outgrow for 12 doublings

678 overnight. The initial populations were verified by phase contrast and fluorescent

679 microscopy. Final ratios were normalized to their starting ratios. Data was plotted using

680 GraphPad Prism.

\section{Microscopy}

682 Phase contrast images of logarithmically growing cells were taken by Zeiss AXIO Scope 683 A1. Cells were mounted on 1\% PYE agar pads and imaged using a 100X objective.

684 MicrobeJ (Ducret et al., 2016) for ImageJ (Schneider et al., 2012) was utilized to 685 quantify cell lengths. Stalk lengths were quantified using ImageJ. Prism was utilized for representations of cell and stalk length measurements. Representative images of the same scale were cropped to display morphological defects.

\section{Protein Purification and Modification}

689 Untagged Lon, untagged CIpX and ClpX*, and his-tagged ClpP were purified as 690 previously described (Chien et al., 2007; Goldberg et al., 1994; Gur and Sauer, 2008;

691 Levchenko et al., 2000). Titin-I27- 320 and Titin-I27-ssrA were purified as previously

692 described (Gur and Sauer, 2008; Kenniston et al., 2003) and labeled with Fluorescein693 5-maleimide (Thermo Scientific ${ }^{\mathrm{TM}}$ ) under guanidine hydrochloride denaturation. The 694 modified protein was buffer exchanged into H-buffer (20 mM Hepes pH 7.5, 100 mM $695 \mathrm{KCl}, 10 \mathrm{mM} \mathrm{MgCl}, 10 \%$ glycerol) and stored at $4^{\circ} \mathrm{C}$. His ${ }_{6} \mathrm{SciP}$ and his 6 CtrA were 696 purified as described in (Gora et al., 2013). DnaA and CcrM were purified as his SUMO 697 tagged proteins, followed by tag cleavage as described (Jonas et al., 2013; Liu et al., 698 2016; Wang et al., 2007). GFP-ssrA was purified as previously described (Yakhnin et 
al., 1998). CtrA-RD+15 was purified as described (Smith et al., 2014). Detailed

purification protocols are available upon request.

\section{In vitro assays}

703 Degradation assays were performed at $30^{\circ} \mathrm{C}$ and monitored using SDS-PAGE gels as 704 described previously (Bhat et al., 2013). The final concentrations used can be found in 705 the figure legends. Densitometry was performed with ImageJ and degradation rates were plotted using Prism.

707

Degradation of FITC-Casein, FT-Titin- $\beta 20$, and FT-Titin-ssrA was monitored as an increase in fluorescence over time. GFP-CtrARD+15 degradation was observed as a loss of fluorescence over time as described previously (Smith et al., 2014).

712 ATP hydrolysis for ClpXP and ClpX*P was measured using a coupled kinase assay as

713 previously described (Burton et al., 2003)

\section{Limited Proteolysis}

716 To perform limited chymotrypsin digestion, $0.004 \mathrm{mg} / \mathrm{mL}$ chymotyrpsin was added to

$7170.01 \mathrm{mg} / \mathrm{mL}$ ClpX or ClpX* in the presence of ClpP and ATP regeneration system and

718 incubated at $25^{\circ} \mathrm{C}$ for indicated time points. To quench the reactions, 5X SDS dye

719 supplemented with $5 \mathrm{mM}$ Protease inhibitor phenylmethylsulfonyl fluoride (PMSF) was

720 added to quench the reactions. The samples were run on a $10 \% \mathrm{Bis}-\mathrm{Tris}$ gel and

721 transferred to nitrocellulose as described above. Membranes were probed with primary

722 antibody in 3\% milk in TBST at $4^{\circ} \mathrm{C}$ overnight with following dilution factors: 1:5000

723 dilution of CIpX. Membranes were washed with 1x TBST for 5 minutes three times and

724 then probed with Licor secondary antibody with 1:10,000 dilution in 1x TBST at room

725 temperature for 1 hour. The protein was visualized using Licor Odyssey CLx. 


\section{Acknowledgements}

731

732

733

734

735

736

737

738

739

740
We thank the Chien, Strieter, Stratton, and Serio lab members for helpful comments and discussion on the manuscript. S.A.M. and B.A. were supported in part through the UMass NIH Chemistry Biology Interface Training Program (NIH T32GM008515) and S.A.M. in part by the HHMI Gilliam Fellowship program. Work in the Chien lab is funded by the NIH (R35GM130320). We thank the Genomics Resource Laboratory (Institute for Applied Life Sciences; University of Massachusetts Amherst) and the Mass Spectrometry Core Facilities for services and equipment support.

Author Contributions: Conceptualization: S.A.M. and P.C.; Investigation, S.A.M. and B.A.; Writing, S.A.M and P.C.; Funding Acquisition, P.C. Supervision, P.C.

Declaration of Interests: The authors declare no competing interests

\section{References}

Baker, T.A., and Sauer, R.T. (2012). CIpXP, an ATP-powered unfolding and proteindegradation machine. Biochim. Biophys. Acta - Mol. Cell Res. 1823, 15-28.

Bhat, N.H., Vass, R.H., Stoddard, P.R., Shin, D.K., and Chien, P. (2013). Identification of ClpP substrates in Caulobacter crescentus reveals a role for regulated proteolysis in bacterial development. 88, 1083-1092.

Breidenstein, E.B.M., Janot, L., Strehmel, J., Fernandez, L., Taylor, P.K., KukavicaIbrulj, I., Gellatly, S.L., Levesque, R.C., Overhage, J., and Hancock, R.E.W. (2012). The Lon Protease Is Essential for Full Virulence in Pseudomonas aeruginosa. PLoS One 7.

Chen, Y.E., Tsokos, C.G., Biondi, E.G., Perchuk, B.S., and Laub, M.T. (2009). Dynamics of two phosphorelays controlling cell cycle progression in Caulobacter crescentus. J. Bacteriol. 191, 7417-7429.

Chien, P., Perchuk, B.S., Laub, M.T., Sauer, R.T., and Baker, T. a (2007). Direct and adaptor-mediated substrate recognition by an essential AAA+ protease. Proc. Natl. Acad. Sci. U. S. A. 104, 6590-6595.

Chowdhury, T., Chien, P., Ebrahim, S., Sauer, R.T., and Baker, T.A. (2010). Versatile modes of peptide recognition by the ClpX N domain mediate alternative adaptor-binding specificities in different bacterial species. Protein Sci. 19, 242-254.

Deatherage, D.E., and Barrick, J.E. (2014). Identification of mutations in laboratory 
evolved microbes from next-generation sequencing data using breseq. Methods Mol Biol. 165-188.

Domian, I.J., Reisenauer, a, and Shapiro, L. (1999). Feedback control of a master bacterial cell-cycle regulator. Proc. Natl. Acad. Sci. U. S. A. 96, 6648-6653.

Doyle, S.M., Shorter, J., Zolkiewski, M., Hoskins, J.R., Lindquist, S., and Wickner, S. (2007). Asymmetric deceleration of ClpB or Hsp104 ATPase activity unleashes proteinremodeling activity. Nat. Struct. Mol. Biol. 14, 114-122.

Ducret, A., Quardokus, E.M., and Brun, Y. V. (2016). MicrobeJ, a tool for high throughput bacterial cell detection and quantitative analysis. Nat. Microbiol. 1, 1-7.

Farrell, C.M., Baker, T.A., and Sauer, R.T. (2007). Altered Specificity of a AAA+ Protease. Mol. Cell 25, 161-166.

Fei, X., Bell, T.A., Barkow, S.R., Baker, T.A., and Sauer, R.T. (2020). Structural basis of clpxp recognition and unfolding of ssra-tagged substrates. Elife 9, 1-39.

Gates, S.N., Yokom, A.L., Lin, J., Jackrel, M.E., Rizo, A.N., Kendsersky, N.M., Buell, C.E., Sweeny, E.A., Mack, K.L., Chuang, E., et al. (2017). Ratchet-like polypeptide translocation mechanism of the AAA+ disaggregase Hsp104. Science (80-. ). 357, 273279.

Goff, S.A., Casson, L.P., and Goldberg, A.L. (1984). Heat shock regulatory gene htpR influences rates of protein degradation and expression of the lon gene in Escherichia coli. Proc. Natl. Acad. Sci. U. S. A. 81, 6647-6651.

Goldberg, A.L. (1972). Degradation of Abnormal Proteins in Escherichia coli. Proc Natl Acad Sci U S A 69, 422-426.

Goldberg, A.L., Moerschell, R.P., Hachung, C., and Maurizi, M.R. (1994). ATPdependent protease La (Lon) from Escherichia coli. Methods Enzymol. 244, 350-375.

Gonzalez, D., Kozdon, J.B., McAdams, H.H., Shapiro, L., and Collier, J. (2014). The functions of DNA methylation by CcrM in Caulobacter crescentus: A global approach. Nucleic Acids Res. 42, 3720-3735.

Gora, K.G., Cantin, A., Wohlever, M., Joshi, K.K., Perchuk, B.S., Chien, P., and Laub, M.T. (2013). Regulated proteolysis of a transcription factor complex is critical to cell cycle progression in Caulobacter crescentus. Mol. Microbiol. 87, 1277-1289.

Gorbatyuk, B., and Marczynski, G.T. (2005). Regulated degradation of chromosome replication proteins DnaA and CtrA in Caulobacter crescentus. Mol. Microbiol. 55, 12331245. 
815 Gottesman, S., Roche, E., Zhou, Y.N., and Sauer, R.T. (1998). The CIpXP and CIpAP

816 proteases degrade proteins with carboxy-terminal peptide tails added by the SsrA-

817 tagging system. Genes Dev. 12, 1338-1347.

818 Gur, E., and Sauer, R.T. (2008). Recognition of misfolded proteins by Lon, a AAA+ protease. Genes Dev. 22, 2267-2277.

Gur, E., Biran, D., and Ron, E.Z. (2011). Regulated proteolysis in Gram-negative bacteria — how and when? Nat Rev Microbiol 9, 839-848.

Asymmetric interactions of ATP with the AAA+ CIpX6 unfoldase: Allosteric control of a protein machine. Cell 121, 1017-1027.

Hottes, A.K., Shapiro, L., and McAdams, H.H. (2005). DnaA coordinates replication initiation and cell cycle transcription in Caulobacter crescentus. Mol. Microbiol. 58, 1340-1353.

Howard-Flanders, P., Simson, E., and Theriot, L. (1964). A locus that controls filament formation and sensitiviry to radiation in Escherichia coli K-12. Genetics 49, 237-246.

Hulsen, T., de Vlieg, J., and Alkema, W. (2008). BioVenn - A web application for the comparison and visualization of biological lists using area-proportional Venn diagrams.

Jenal, U., and Fuchs, T. (1998). An essential protease involved in bacterial cell-cycle

Jonas, K., Liu, J., Chien, P., and Laub, M.T. (2013). Proteotoxic stress induces a cellcycle arrest by stimulating lon to degrade the replication initiator DnaA. Cell 154, 623636. 431.

Keiler, K.C. (2015). Mechanisms of ribosome rescue in bacteria. Nat. Rev. Microbiol. 13, 285-297.

Keiler, K.C., Waller, P.R.H., and Sauer, R.T. (1996). Role of a Peptide Tagging System 990-993. an AAA+ degradation machine. Cell 114, 511-520. 
861 Kim, D.Y., and Kim, K.K. (2003). Crystal Structure of ClpX Molecular Chaperone from

862 Helicobacter pylori. J. Biol. Chem. 278, 50664-50670.

863

864

865

866

867

868

869

870

871

872

873

874

875

876

877

878

879

880

881

882

883

884

885

886

887

888

889

890

891

892

893

894

895

896

897

898

899

900

901

902

903

904

905

906

Langklotz, S., Baumann, U., and Narberhaus, F. (2012). Structure and function of the bacterial AAA protease FtsH. Biochim. Biophys. Acta - Mol. Cell Res. 1823, 40-48.

Laub, M.T., Chen, S.L., Shapiro, L., and McAdams, H.H. (2002). Genes directly controlled by CtrA, a master regulator of the Caulobacter cell cycle. Proc. Natl. Acad. Sci. U. S. A. 99, 4632-4637.

Lee, I., and Suzuki, C.K. (2008). Functional mechanics of the ATP-dependent Lon protease- lessons from endogenous protein and synthetic peptide substrates. Biochim. Biophys. Acta - Proteins Proteomics 1784, 727-735.

Lee, S., Roh, S.H., Lee, J., Sung, N., Liu, J., and Tsai, F.T.F. (2019). Cryo-EM Structures of the Hsp104 Protein Disaggregase Captured in the ATP Conformation. Cell Rep. 26, 29-36.e3.

Levchenko, I., Seidel, M., Sauer, R.T., and Baker, T.A. (2000). A Specificity-Enhancing Factor for the CIpXP Degradation Machine. Science (80-. ). 289, 2354-2356.

Li, H., and Durbin, R. (2009). Fast and accurate short read alignment with BurrowsWheeler transform. Bioinformatics 25, 1754-1760.

Li, H., Handsaker, B., Wysoker, A., Fennell, T., Ruan, J., Homer, N., Marth, G., Abecasis, G., and Durbin, R. (2009). The Sequence Alignment/Map format and SAMtools. Bioinformatics 25, 2078-2079.

Liu, J., Francis, L.I., Jonas, K., Laub, M.T., and Chien, P. (2016). CIpAP is an auxiliary protease for DnaA degradation in Caulobacter crescentus. Mol. Microbiol. 102, 10751085.

Lopez, K.E., Rizo, A.N., Tse, E., Lin, J.B., Scull, N.W., Thwin, A.C., Lucius, A.L., Shorter, J., and Southworth, D.R. (2020). Conformational plasticity of the CIpAP AAA+ protease couples protein unfolding and proteolysis. Nat. Struct. Mol. Biol. 27, 406-416.

Mahmoud, S.A., and Chien, P. (2018). Regulated Proteolysis in Bacteria. Annu. Rev. Biochem. 87, 677-696.

Martin, A., Baker, T.A., and Sauer, R.T. (2008). Protein unfolding by a AAA+ protease is dependent on ATP-hydrolysis rates and substrate energy landscapes. Nat. Struct. Mol. Biol. 15, 139-145.

Mizusawa, S., and Gottesman, S. (1983). Protein degradation in Escherichia coli: the lon gene controls the stability of sulA protein. Proc. Natl. Acad. Sci. U. S. A. 80, 358362 . 
Persat, A., Stone, H.A., and Gitai, Z. (2014). The curved shape of caulobacter crescentus enhances surface colonization in flow. Nat. Commun. 5, 1-9. Quinlan, A.R., and Hall, I.M. (2010). BEDTools: a flexible suite of utilities for comparing genomic features. Bioinformatics 26, 841-842.

Quon, K.C., Marczynski, G.T., and Shapiro, L. (1996). Cell cycle control by an essential bacterial two-component signal transduction protein. Cell 84, 83-93.

R Core Team (2019). R: A language and Environment for Statistical Computing (R Foundation for Statistical Computing).

Ripstein, Z.A., Vahidi, S., Houry, W.A., Rubinstein, J.L., and Kay, L.E. (2020). A processive rotary mechanism couples substrate unfolding and proteolysis in the CIpXP degradation machinery. Elife 9.

Robinson, M.D., McCarthy, D.J., and Smyth, G.K. (2009). edgeR: A Bioconductor package for differential expression analysis of digital gene expression data.

Bioinformatics 26, 139-140.

Rogers, A., Townsley, L., Gallego-hernandez, A.L., Beyhan, S., Kwuan, L., and Yildiz, F.H. (2016). and the Type VI Secretion System in Vibrio cholerae. 198, 973-985.

Sauer, R.T., and Baker, T.A. (2011). AAA+ Proteases: ATP-Fueled Machines of Protein Destruction. Annu. Rev. Biochem 80, 587-612.

Schneider, C.A., Rasband, W.S., and Eliceiri, K.W. (2012). NIH Image to ImageJ: 25 years of image analysis. Nat. Methods 9, 671-675.

Shin, M., Puchades, C., Asmita, A., Puri, N., Adjei, E., Luke Wiseman, R., Wali Karzai, A., and Lander, G.C. (2020). Structural basis for distinct operational modes and protease activation in AAA+ protease Lon.

Skerker, J.M., Prasol, M.S., Perchuk, B.S., Biondi, E.G., and Laub, M.T. (2005). Twocomponent signal transduction pathways regulating growth and cell cycle progression in a bacterium: A system-level analysis. PLoS Biol. 3.

Smith, S.C., Joshi, K.K., Zik, J.J., Trinh, K., Kamajaya, A., Chien, P., and Ryan, K.R. (2014). Cell cycle-dependent adaptor complex for ClpXP-mediated proteolysis directly integrates phosphorylation and second messenger signals. Proc. Natl. Acad. Sci. U. S. A. 111, 14229-14234.

Tan, H.M., Kozdon, J.B., Shen, X., Shapiro, L., and McAdams, H.H. (2010). An essential transcription factor, SciP, enhances robustness of Caulobacter cell cycle regulation. Proc. Natl. Acad. Sci. U. S. A. 107, 18985-18990. 
953 Wang, K.H., Sauer, R.T., and Baker, T.A. (2007). ClpS modulates but is not essential

954 for bacterial N-end rule degradation. Genes Dev. 21, 403-408.

955

956

957

958

959

960

961

962

963

964

965

966

967

968

969

970

971

972

973

974

975

976

977

978

979

980

981

982

983

984

985

986

987

988

989

990

991

992

993

994

995

996

997

998

Witkin, E.M. (1946). Inherited Differences in Sensitivity to Radiation in Escherichia Coli. Proc. Natl. Acad. Sci. U. S. A. 32, 59-68.

Wortinger, M., Sackett, M.J., and Brun, Y. V. (2000). CtrA mediates a DNA replication checkpoint that prevents cell division in Caulobacter crescentus. EMBO J. 19, 45034512.

Wright, R., Stephens, C., Zweiger, G., Shapiro, L., and Alley, M.R.K. (1996).

Caulobacter Lon protease has a critical role in cell-cycle control of DNA methylation. Genes Dev. 10, 1532-1542.

Wu, D., Lim, E., Vaillant, F., Asselin-Labat, M.L., Visvader, J.E., and Smyth, G.K. (2010). ROAST: Rotation gene set tests for complex microarray experiments. Bioinformatics 26, 2176-2182.

Yakhnin, A. V., Vinokurov, L.M., Surin, A.K., and Alakhov, Y.B. (1998). Green fluorescent protein purification by organic extraction. Protein Expr. Purif. 14, 382-386.

Ye, X., Lin, J., Mayne, L., Shorter, J., and Walter Englander, S. (2020). Structural and kinetic basis for the regulation and potentiation of Hsp104 function. Proceeding Natl. Acad. Sci. .

Yokom, A.L., Gates, S.N., Jackrel, M.E., Mack, K.L., Su, M., Shorter, J., and Southworth, D.R. (2016). Spiral architecture of the Hsp104 disaggregase reveals the basis for polypeptide translocation. Nat. Struct. Mol. Biol. 23, 830-837.

Zeinert, R.D., Liu, J., Yang, Q., Du, Y., Haynes, C.M., and Chien, P. (2018). A legacy role for DNA binding of Lon protects against genotoxic stress. BioRxiv. 


\section{Supplemental Figure Legends}

\section{Figure S1: Validation of motility screen suppressor}

(A-B) Motility assay on $0.3 \%$ PYE agar of indicated strains.

C. Sequence alignment of $\mathrm{ClpX}$ homologs. Conserved glycine residue and walker B motif are marked.

D. Quantification of the percent of cells with DNA content of $1 \mathrm{~N}, 2 \mathrm{~N}$, and $>2 \mathrm{~N}$ in $w t$, $\Delta l o n$, and $\Delta$ lon $c / p X^{*}$ strains.

\section{Figure S2: RNA-seq gene set analysis}

(A-B) Volcano plot showing the transcriptional differences of $\Delta$ lon compared to wt (left) and $\Delta$ lon clpX* compared to $w t$ (right), as measured by RNA-seq. The negative $\log _{10}$ of the $p$ value is plotted against $\log _{2}$ of the fold change mRNA counts. Genes that are marked in red have an FDR $<0.05$. Transcripts that are associated with either the SciP regulon (Tan et al., 2010) or cell cycle (KEGG pathways) are highlighted in blue. C. Kyoto Encyclopedia of Genes and Genomes (KEGG) pathway enrichment analysis and FRY gene set analysis. FRY gene set analysis performed using fry function from edgeR package in $\mathrm{R}$ (Robinson et al., 2009; Wu et al., 2010). The CcrM, DnaA, and SciP regulons were assigned according to (Gonzalez et al., 2014; Hottes et al., 2005; Tan et al., 2010). Over-represented pathways in KEGG pathway database identified using the kegga function from edge $R$ package in $\mathrm{R}$.

\section{Figure S3: $c / p X^{*}$ mutant is dominant}

A. Western blot showing CcrM levels in synchronized populations of wt, $4 / 0 n$, and $\Delta / o n$ $c / p X^{*}$ cells. Swarmer cells were isolated using a density gradient and an equal number of cells were released into fresh PYE medium. Samples were withdrawn at the indicated time points and probed with anti-CcrM.

B. Growth curves of wild type (wt), $\Delta / O$ n, and $\Delta / o n$ merodiploid with second copy of $c / p X^{*}$ at native locus. Cells grown in PYE.

C. Spot assays comparing colony formation of strains in PYE and PYE supplemented with mitomycin C.

D. Motility assay on $0.3 \%$ PYE agar of indicated strains

\section{Figure S4: In vitro characterization of CIpX*}

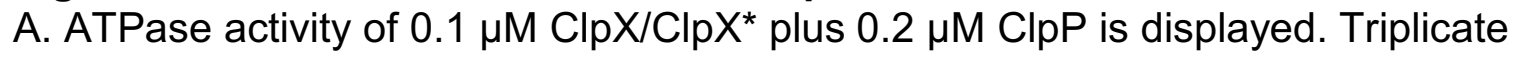
experiments are shown.

B. In vitro degradation of CcrM by $0.2 \mu \mathrm{M}$ Lon is shown as a control. The bottom gel shows CcrM degradation assay in the presence of ATP, with no regeneration mix. C. FITC-Casein titration in the presence of either ClpXP or ClpX*P. Data was fit to the Michaelis-Menten equation. ClpX*P degrades FITC-Casein 2-3 fold better than CIpXP. Inset shows residual plot for Michaelis-Menten fit F. Fluorescin-Titin-I27- 320 titration in the presence of either ClpXP or ClpX*P. Data was fit to the Michaelis-Menten equation. $V_{\text {max }} / K_{M}$ was $30 \%$ higher for $C l p{ }^{*} P$ in comparison to ClpXP. Inset shows residual plot for Michaelis-Menten fit. 
1045 Figure S5 ClpX*P is deficient in native substrate degradation

1046 A. In vitro degradation of GFP-ssrA.

1047 B. Fluorescein-Titin-I27-ssrA titration in the presence of either ClpXP or CIpX*P. Data was fit to the Michaelis-Menten equation. $V_{\text {max }} / K_{M}$ was $30 \%$ lower for $C l p X^{*} P$ in comparison to ClpXP. Inset shows residual plot for Michaelis-Menten fit. C. In vitro fluorescence degradation assay of eGFP-CtrA-RD+15 in the presence of adaptors. Degradation assays were performed with $1 \mu \mathrm{M}$ eGFP-CtrA-RD+15, $0.1 \mu \mathrm{M}$ ClpX or ClpX* hexamer, $0.2 \mu \mathrm{M}$ ClpP, $2 \mu \mathrm{M}$ CpdR, $1 \mu \mathrm{M}$ RcdA, $1 \mu \mathrm{M}$ PopA, and $20 \mu \mathrm{M}$ cyclic di-GMP, and ATP regeneration system. Assays were performed with $0.1 \mu \mathrm{M}$ ClpX or ClpX* hexamer, $0.2 \mu \mathrm{M}$ ClpP, ATP regeneration system, and $10 \mu \mathrm{M}$ GFP-ssrA. B. In vitro degradation of SciP by CIpXP under low and saturating ATP conditions. Assays were performed with $0.1 \mu \mathrm{M} \mathrm{ClpX}_{6}$ or $\mathrm{ClpX}_{6}{ }^{*}$, and $0.2 \mu \mathrm{M} \mathrm{ClpP}_{14}$ and $5 \mu \mathrm{M}$ SciP. Quantification of triplicate experiments shown. 
bioRxiv preprint doi: https://doi.org/10.1101/2021.08.18.456811; this version posted August 18, 2021. The copyright holder for this preprint (which was not certified by peer review) is the author/funder, who has granted bioRxiv a license to display the preprint in perpetuity. It is made available under aCC-BY-NC-ND 4.0 International license.

Figure S1 - related to figure 1

A.

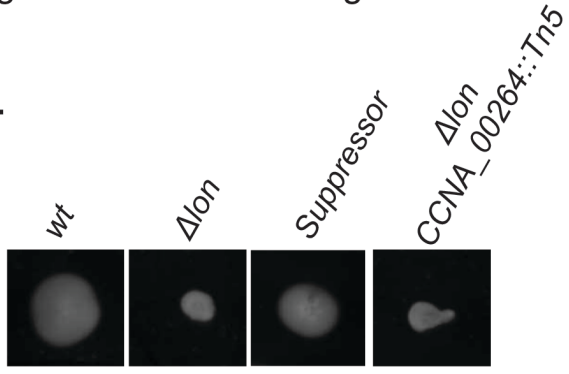

C.

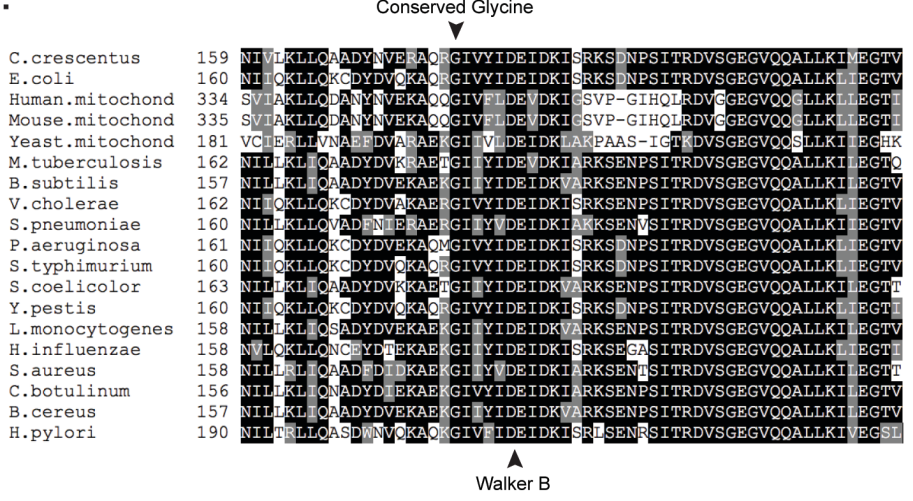

B.

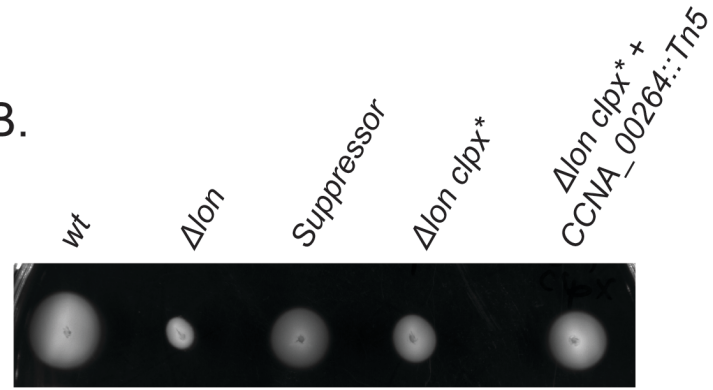

D.

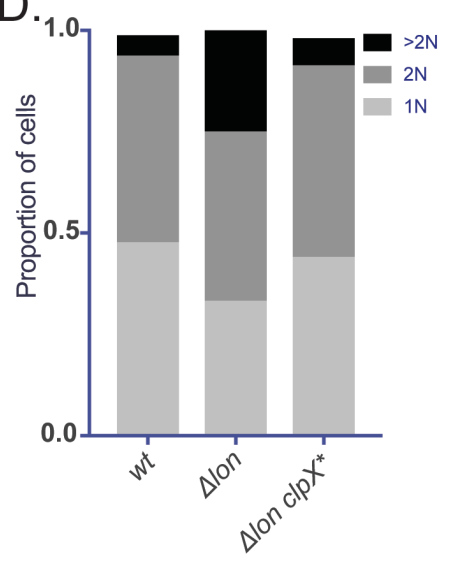


bioRxiv preprint doi: https://doi.org/101101/2021.08.18.456811. this version posted August 18,2021 . The copyright holder for this preprint (which was not certified by peer review) is the author/funder, who has granted bioRxiv a license to display the preprint in perpetuity. It is made available under aCC-BY-NC-ND 4.0 International license.

Figure S2- Related to Figure 2

A. $\circ$ FDR $<0.05$

- SciP Regulon/Cell Cycle

\section{SciP Regulon}

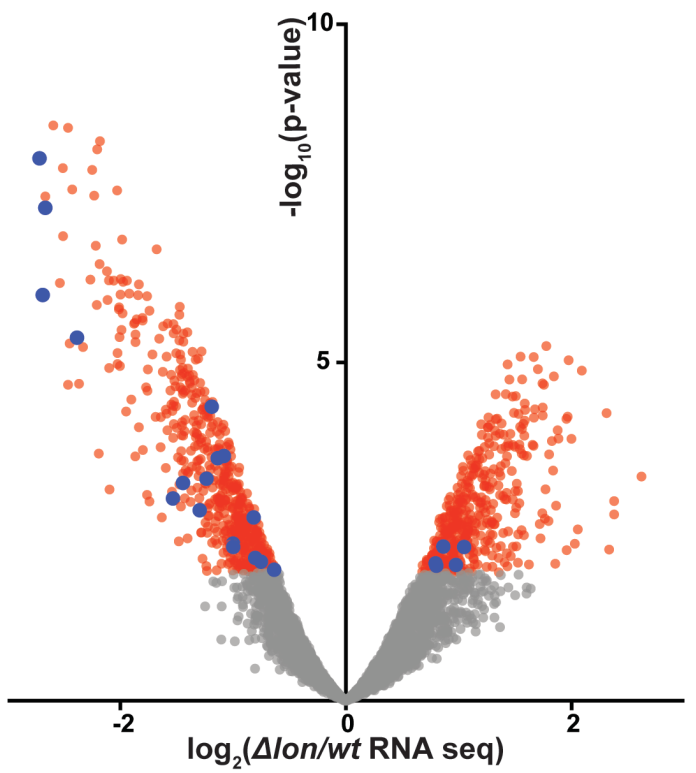

B.

.
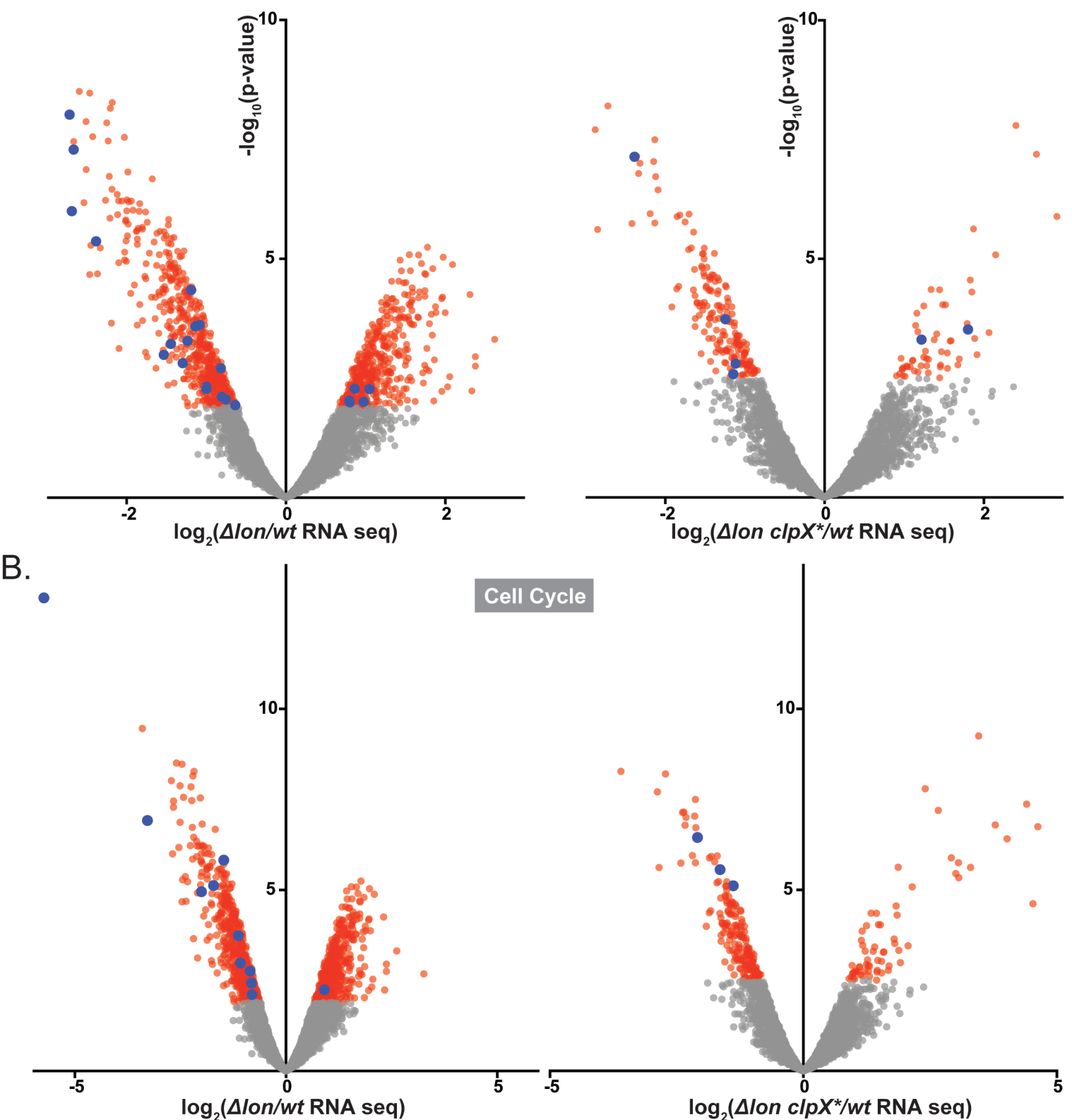

C.

FRY Gene Set Analysis

\begin{tabular}{|c|c|c|c|c|c|c|c|c|}
\hline & \multicolumn{4}{|c|}{$\Delta$ lon vs wt } & \multicolumn{4}{|c|}{$\Delta$ lon clpX* vs wt } \\
\hline & $\begin{array}{c}\text { Number of } \\
\text { Genes }\end{array}$ & Direction & $p$-value & FDR & $\begin{array}{c}\text { Number of } \\
\text { Genes }\end{array}$ & Direction & $\mathrm{p}$-value & FDR \\
\hline SciP & 61 & Down & 0.0029 & 0.0044 & 61 & Down & 0.3391 & 0.5042 \\
\hline CcrM & 504 & Up & 0.0014 & 0.0014 & 504 & Up & 0.7391 & 0.7391 \\
\hline DnaA & 32 & Down & 0.0989 & 0.0980 & 32 & Down & 0.0919 & 0.1275 \\
\hline
\end{tabular}

$\log _{2}\left(\Delta / o n\right.$ clp $X^{*} /$ wt RNA seq)

\begin{tabular}{|c|c|c|c|}
\hline & \multicolumn{3}{|c|}{ Cell Cycle } \\
\hline & $\begin{array}{c}\text { Number of } \\
\text { Genes }\end{array}$ & Direction & p-value \\
\hline$\Delta$ lon vs wt & 31 & 10, Down & 0.0086 \\
\hline $\begin{array}{c}\Delta \text { Ilon clp } X^{*} \\
\text { vs wt }\end{array}$ & 31 & 3, Down & 0.1725 \\
\hline
\end{tabular}


Figure S3- Related to Figure 2

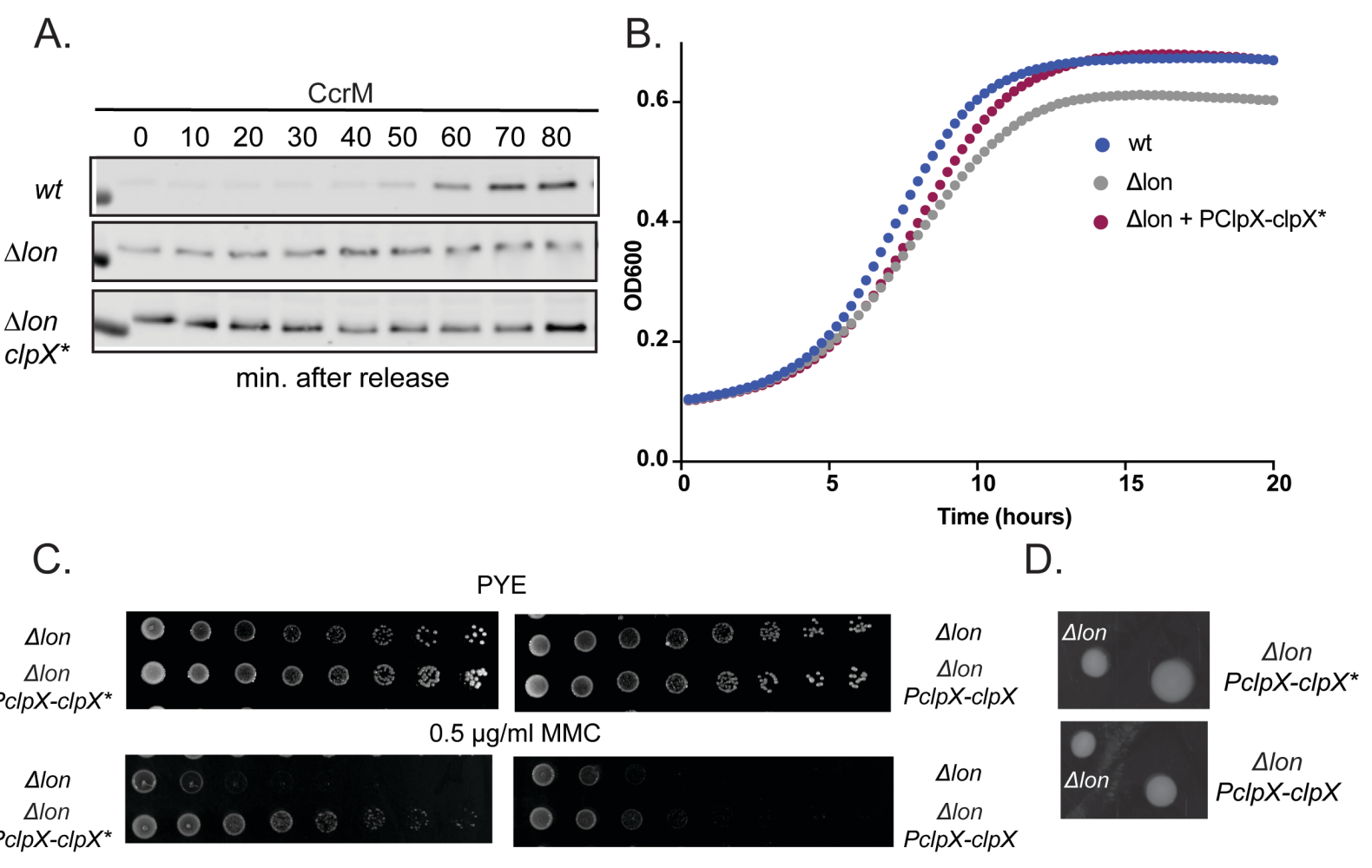


Figure S4- Related to Figure 3
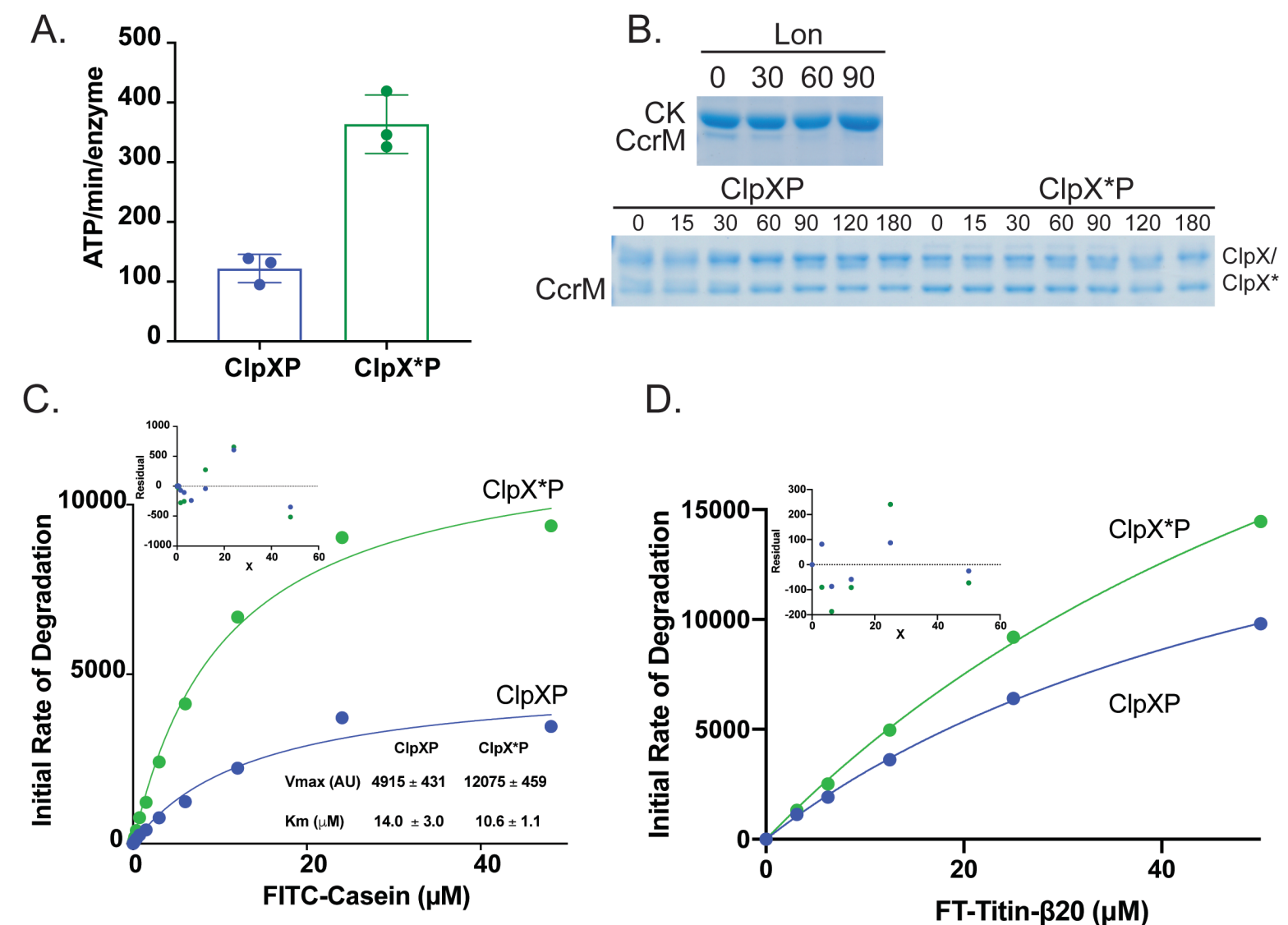

D.

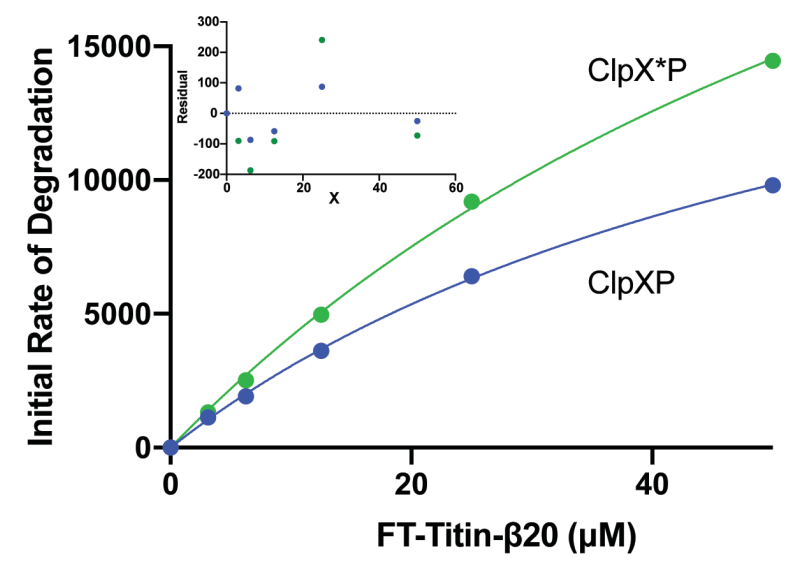


Figure S5- Related to Figure 4

A.

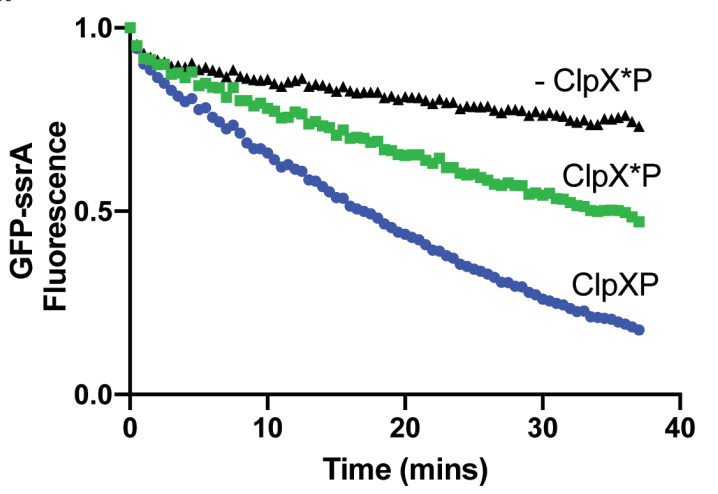

C.

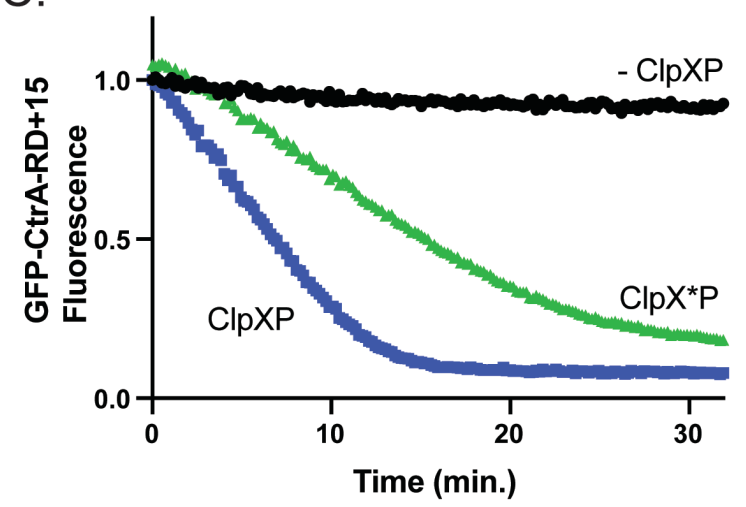

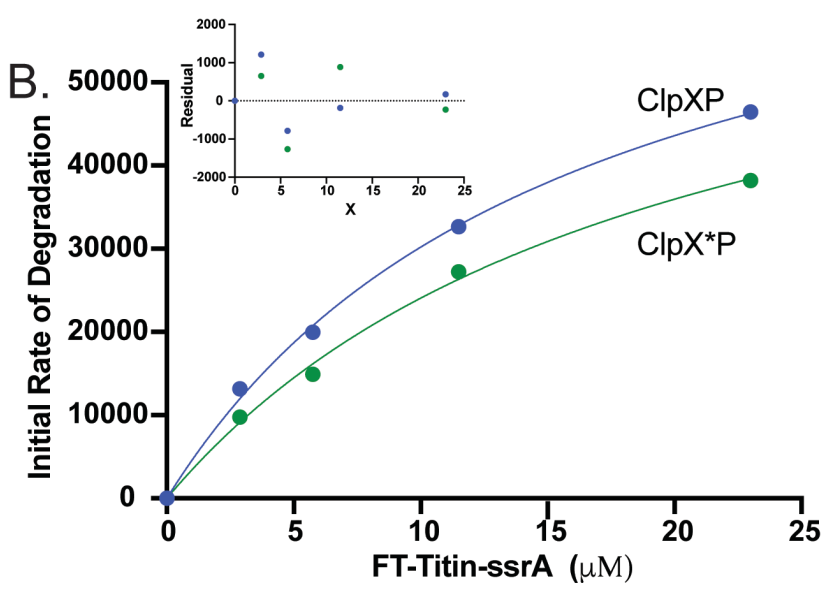




\section{Figure S6- Related to Figure 5}

A.

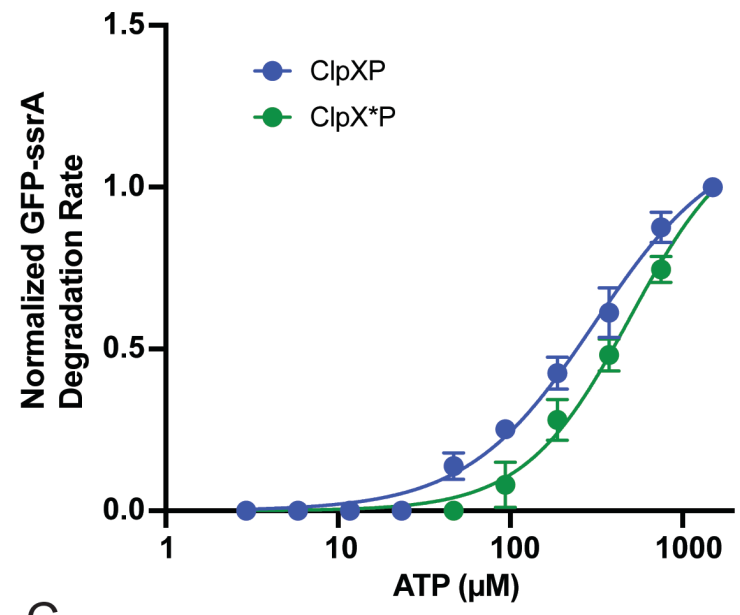

C.

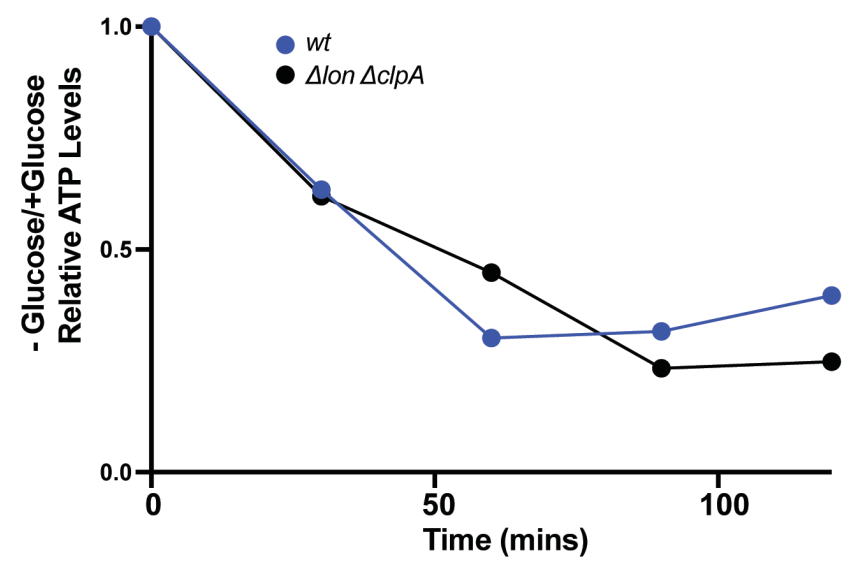

B.

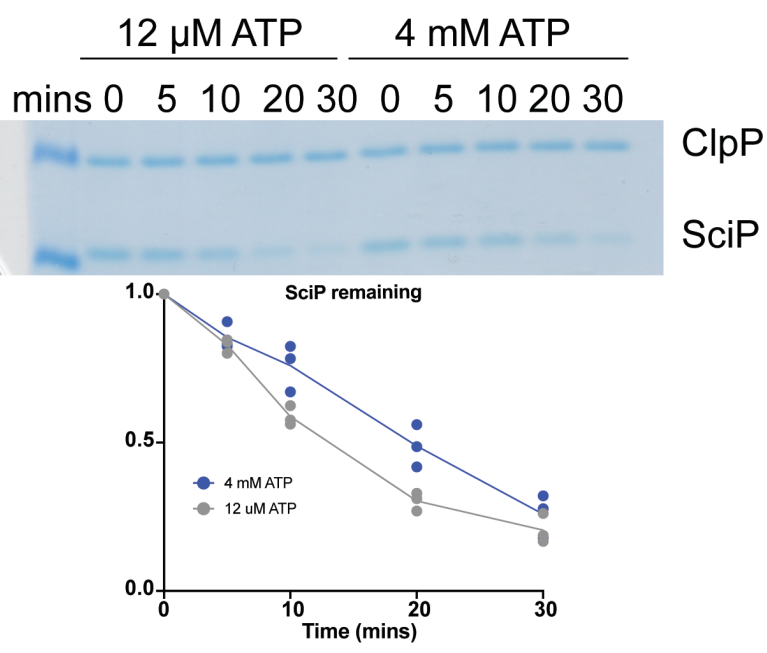

